\title{
High-resolution transmission spectroscopy of MASCARA-2 b with EXPRES
}

\author{
H. Jens Hoeijmakers ${ }^{1,2}$, Samuel H. C. Cabot ${ }^{3}$, Lily Zhao ${ }^{3}$, Lars A. Buchhave ${ }^{4}$, René Tronsgaard ${ }^{4}$, \\ Allen B. Davis ${ }^{3}$, Daniel Kitzmann ${ }^{2}$, Simon L. Grimm ${ }^{2}$, Heather M. Cegla ${ }^{1}$, Vincent Bourrier ${ }^{1}$, \\ David Ehrenreich $^{1}$, Kevin Heng ${ }^{2,5}$, Christophe Lovis ${ }^{1}$, and Debra A. Fischer ${ }^{3}$
}

\author{
${ }^{1}$ Observatoire de Genève, Chemin des Maillettes 51, 1290 Versoix, Switzerland \\ e-mail: jens.hoeijmakers@unige.ch \\ ${ }^{2}$ Center for Space and Habitability, Universität Bern, Gesellschaftsstrasse 6, 3012 Bern, Switzerland \\ ${ }^{3}$ Yale University, 52 Hillhouse, New Haven, CT 06511, USA \\ ${ }^{4}$ DTU Space, National Space Institute, Technical University of Denmark, Elektrovej 328, 2800 Kgs. Lyngby, Denmark \\ ${ }^{5}$ University of Warwick, Department of Physics, Astronomy \& Astrophysics Group, Coventry CV4 7AL, UK
}

Received 2 January 2020 / Accepted 17 April 2020

\begin{abstract}
We report detections of atomic species in the atmosphere of MASCARA-2 b, using the first transit observations obtained with the newly commissioned EXPRES spectrograph. EXPRES is a highly stabilized optical echelle spectrograph, designed to detect stellar reflex motions with amplitudes down to $30 \mathrm{~cm} \mathrm{~s}^{-1}$, and has recently been deployed at the Lowell Discovery Telescope. By analyzing the transmission spectrum of the ultra-hot Jupiter MASCARA-2 b using the cross-correlation method, we confirm previous detections of $\mathrm{Fe}$ I, Fe II, and Na I, which likely originate in the upper regions of the inflated atmosphere. In addition, we report significant detections of $\mathrm{Mg}$ I and CrII. The absorption strengths change slightly with time, possibly indicating different temperatures and chemistry in the day- and nightside terminators. Using the effective stellar line-shape variation induced by the transiting planet, we constrain the projected spin-orbit misalignment of the system to $1.6 \pm 3.1$ degrees, consistent with an aligned orbit. We demonstrate that EXPRES joins a suite of instruments capable of phase-resolved spectroscopy of exoplanet atmospheres.
\end{abstract}

Key words. instrumentation: spectrographs - planets and satellites: atmospheres - techniques: spectroscopic planets and satellites: gaseous planets

\section{Introduction}

The emergence of a new generation of spectrographs opens avenues for detecting and characterizing exoplanets and their atmospheres with unprecedented fidelity. Exoplanets can be detected with high-resolution spectrographs due to their gravitational interaction with the host star, causing a periodic Doppler shift in the observed stellar spectrum as the planet orbits. These measurements yield a lower limit to the mass of the planet or the mass itself if the planet orbits in an aligned plane that carries it through transit as seen from Earth. The composition of a transiting planet may be constrained by also measuring its radius, which then yields the mean density. Recent years have seen extensive efforts to develop a new generation of environmentally controlled high-resolution spectrographs that provide ever increasing radial velocity $(\mathrm{RV})$ precision and stability, to allow for more precise mass measurements and for the detection of less massive planets farther away from their host star. Indeed, several purpose-built instruments have come online in recent years. ESPRESSO ${ }^{1}$ (Pepe et al. 2013) was commissioned at the Very Large Telescope (VLT) in December 2017. HARPS-N/GIANO ${ }^{2}$ (Cosentino et al. 2012; Claudi et al. 2017; Oliva et al. 2018) at the Telescopio Nazionale Galileo (TNG),

1 Echelle SPectrograph for Rocky Exoplanets and Stable Spectroscopic Observations.

2 High Accuracy Radial velocity Planet Searcher-North.
CARMENES $^{3}$ (Quirrenbach et al. 2010) at Calar Alto Observatory, and SPIRou ${ }^{4}$ (Thibault et al. 2012) at the Canada France Hawaii Telescope (CFHT) provide high-resolution optical and near-IR (NIR) coverage. NIRPS ${ }^{5}$ (Wildi et al. 2017) at the ESO $3.6 \mathrm{~m}$ telescope at La Silla and HARPS3 (Thompson et al. 2016) at the Isaac Newton Telescope are currently under development.

The EXtreme PREcision Spectrograph (EXPRES) was commissioned at the Lowell Observatory $4.3 \mathrm{~m}$ Lowell Discovery Telescope (LDT, Levine et al. 2012) in 2018. EXPRES is a vacuum-stabilized, fiber-fed, $R \sim 140000$, optical spectrograph with wavelength calibration from a Menlo Systems laser frequency comb (LFC). It is optimized for wavelengths between 380 and $680 \mathrm{~nm}$. The design goal of EXPRES was an RV precision of $\sim 30 \mathrm{~cm} \mathrm{~s}^{-1}$ on bright $(V<8)$ main-sequence stars. Observations with the LFC demonstrate an instrumental stability of $7 \mathrm{~cm} \mathrm{~s}^{-1}$ and formal errors of about $25 \mathrm{~cm} \mathrm{~s}^{-1}$ for a signal-tonoise ratio $(\mathrm{S} / \mathrm{N})$ of 250 when observing bright stars (Blackman et al. 2019; Petersburg et al. 2020).

High-resolution spectrographs are able to directly probe spectral features in the atmospheres of exoplanets (Snellen et al. 2008). The high spectral resolution allows for individual spectral

\footnotetext{
Calar Alto high-Resolution search for M dwarfs with Exoearths with Near-infrared and optical Echelle Spectrographs.

4 SPectropolarimètre InfraROUge.

5 Near Infra-Red Planet Searcher.
} 
lines in the planetary spectrum to be resolved and provides measurements of the line shape and depth (e.g., Redfield et al. 2008; Wyttenbach et al. 2015, 2017; Jensen et al. 2012; Khalafinejad et al. 2017; Allart et al. 2019; Seidel et al. 2019). The orbital velocity of hot Jupiters is often in excess of $100 \mathrm{~km} \mathrm{~s}^{-1}$. At $R \sim 10^{5}$, this motion is resolved in time-series exposures of a several-hour transit. Separating the spectrum of the planet from the host star using this Doppler shift was first applied to detect absorption by atmospheric $\mathrm{CO}$ in the infrared transmission spectrum of HD 209458 b (Snellen et al. 2010). Later efforts employed this technique to detect the thermal emission of both transiting and nontransiting hot Jupiters, resulting in detections of $\mathrm{CO}, \mathrm{H}_{2} \mathrm{O}, \mathrm{CH}_{4}$, and $\mathrm{HCN}$ (e.g., Brogi et al. 2012, 2016; Birkby et al. 2013, 2017; Lockwood et al. 2014; Piskorz et al. 2016; Hawker et al. 2018; Cabot et al. 2019; Guilluy et al. 2019; Flagg et al. 2019). The technique has also been applied at optical wavelengths to detect $\mathrm{TiO}$ in the dayside spectrum of WASP33 b using the HDS/Subaru ${ }^{6}$ instrument (Nugroho et al. 2017) and atomic metal lines in the transmission spectrum of KELT9 b (Hoeijmakers et al. 2018a, 2019) using HARPS-N/TNG and PEPSI/LBT ${ }^{7}$ (Cauley et al. 2019). Ongoing improvements in resolution, stability, and wavelength calibration will enable more detailed detections of chemistry and atmospheric dynamics for a multitude of exoplanet systems (Heng \& Showman 2015; Crossfield 2015; Madhusudhan et al. 2016; Birkby 2018; Triaud 2018; Wright 2018). Stabilized fiber-fed high-resolution spectrographs thus have a complementary dual-use in the discovery of exoplanets as well as in the characterization of their atmospheres.

MASCARA-2 b/Kelt-20 b (hereafter M2) was independently discovered by the MASCARA ${ }^{8}$ (Talens et al. 2018) and KELT ${ }^{9}$ (Lund et al. 2017) instruments, which both survey bright stars for signs of periodically transiting exoplanets. The planet is a hot Jupiter, transiting the bright $\left(m_{V}=7.6\right)$ A2 main-sequence star HD 185603 in a 3.47 day orbit. The orbit of the planet is aligned, which is not common for this type of star (Winn et al. 2010; Schlaufman 2010; Albrecht et al. 2012). Strong irradiation from its host star gives M2 b a high equilibrium temperature of $T_{\text {eq }} \sim 2260 \mathrm{~K}$, placing it in the extreme class of ultrahot Jupiters. Several such planets have been studied to date, most notably, KELT-9 b, which with $T_{\text {eq }}=4050 \mathrm{~K}$ is the hottest known planet around a main-sequence star. In light of previous detections of atomic metals in KELT-9 b (Hoeijmakers et al. 2018a, 2019), atmospheric studies of other ultrahot Jupiters warrant searches for vaporized metals and ions.

The transmission spectrum of M2 has been observed extensively with the HARPS-N and CARMENES spectrographs, leading to detections of two Balmer lines of hydrogen, the $\mathrm{Na} I$ D-lines, the Ca II IR triplet, Fe I, Fe II, and tentative evidence for an Mg I line at 517.268 (Casasayas-Barris et al. 2018, 2019; Nugroho et al. 2020; Stangret et al. 2020). These detections are indicative of the high temperatures prevalent on the dayside and in terminator regions. The detection of strong hydrogen absorption may further be indicative of an extended or evaporating atmosphere (Yan \& Henning 2018; Turner et al. 2020).

This paper presents the results of one night of transit observations of M2 with the EXPRES instrument, which constitute the first application of EXPRES for the purpose of exoplanet transit transmission spectroscopy. With these observations, we aim to

\footnotetext{
6 High Dispersion Spectrograph.

Potsdam Echelle Polarimetric and Spectroscopic Instrument, Large Binocular Telescope.

8 Multi-site All-Sky CAmeRA.

9 Kilo-degree Extremely Little Telescope.
}

demonstrate that EXPRES offers significant potential for atmospheric characterization in addition to its main purpose of RV monitoring for exoplanet discovery. The paper is organized as follows: in Sect. 2 we describe the observations and present our analysis of the transmission spectra. In Sect. 3 we discuss our results, including an analysis of the Doppler shadow induced by the Rossiter-McLaughlin (RM) effect. We detect spectroscopic signatures of the atmosphere of M2 by confirming the presence of Fe I and Fe II (Casasayas-Barris et al. 2019; Nugroho et al. 2020; Stangret et al. 2020), and we present strong evidence for $\mathrm{Mg}$ I, Na I, and Cr II. We summarize our results in Sect. 4.

\section{Transit observations of MASCARA-2b}

A single transit of M2 was observed with the LDT during the night of June 1, 2018. The night was clear with an average seeing of approximately 1 .' 2 . The observations lasted from 09:03 to 14:23 UTC, with a total of 68 exposures of the system, of which 51 were obtained in-transit (we include ingress and egress in our in-transit sample). The $\mathrm{S} / \mathrm{N}$ for these exposures reached approximately 40-50 near $5000 \AA$ throughout the night. Most exposures were approximately $200 \mathrm{~s}$, while 15 -min exposures at the start of the night provided out-of-transit spectra with a high $\mathrm{S} / \mathrm{N}$.

Wavelength calibration was carried out with a Menlo Systems LFC as well as with a ThAr line-lamp. Exposures were taken through the science fiber and were interspersed with science observations approximately every $30 \mathrm{~min}$. A tuneable LED was used to take flat-fields. Sets of zero-second bias frames were taken before and after the observing night, and dark current was corrected using the CCD overscan regions. We performed extractions with the RePack ${ }^{10}$ code, adapted from a version purposed for use with HARPS-N data products (Fischer et al. 2016). We also divided spectra by a blaze-function derived from flatfield calibrations. The result is a pipeline-reduced spectral time series in Earth's rest-frame, shown in the top panel of Fig. 1.

Our subsequent analysis follows the approach of Hoeijmakers et al. (2019). This approach resulted in the detections of iron and titanium in the atmosphere of KELT-9 b, using a similar sequence of observations by the HARPS-N spectrograph. The cross-correlation procedure discussed below differs from the approach used by Casasayas-Barris et al. (2018, 2019), which was based on the direct analysis of individual absorption lines in a single coadded transmission spectrum.

\subsection{Telluric correction and detrending}

Absorption lines from the stellar photosphere as well as the Earth's atmosphere (telluric lines) are the most dominant spectral features in the observed spectra. We modeled the telluric contamination in each spectrum with the Molecfit package (Smette et al. 2015). Molecfit processes an observed spectrum along with ambient weather, time, and location information to model the structure of the Earth's atmosphere during an observation and to fit a line-by-line radiative transfer model to the spectrum. We used similar initial parameters as Allart et al. (2017), such as the atmospheric profile, degree of the continuum, and wavelength polynomial fits, and resolution fit kernel. We chose more relaxed convergence tolerances of $10^{-5}$, which improved run time and the quality of fit. Because M2 is a fast rotator, most stellar lines are significantly broadened and blended to form a quasi-continuum, minimizing their effect on the fit of individual telluric lines. We fit each spectrum in the time

$\overline{10}$ Written by Lars Buchhave. 

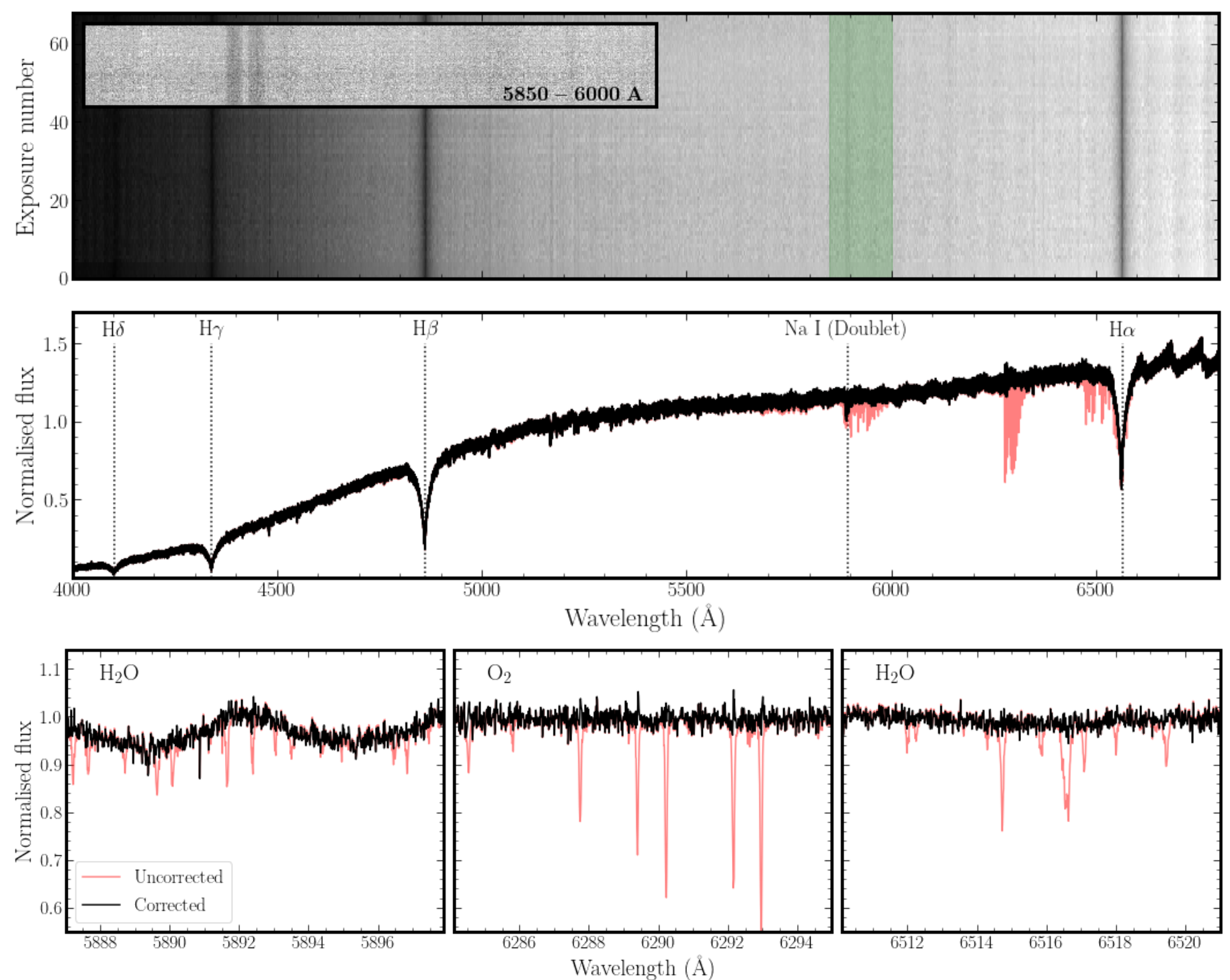

Fig. 1. Spectra obtained on June 1, 2018, with EXPRES. Top panel: time-series, high-resolution spectra cleaned and corrected for tellurics. The orders are stitched together for visualization, but are analyzed independently during cross-correlation. Common features such as stellar lines appear as dark columns. Inset: zoom-in of the time-series spectra between 5850 and $6000 \AA$ (marked by the green band), revealing the broadened Na doublet. Middle panel: example 1D spectrum from the time series before (red) and after (black) telluric correction. The features around $6700 \AA$ are an artifact of order stitching. Some strong stellar lines, including the Balmer series and the sodium D lines, are marked. Bottom panels: zoom-in of the example 1D spectrum, demonstrating the effect of telluric correction in strongly contaminated regions (contaminated by $\mathrm{H}_{2} \mathrm{O}, \mathrm{O}_{2}$, and $\mathrm{H}_{2} \mathrm{O}$, from left to right).

series individually and divided the spectrum by the fit to remove tellurics (Fig. 1, middle and bottom panels).

Our analysis used spectra in the wavelength range 4000$6800 \AA$ A. Bluer wavelengths are subject to very low instrumental throughput, and redder wavelengths suffer severe telluric contamination that is more difficult to model while covering comparatively few atomic metal lines. This left 64 out of 88 orders, which were treated separately until their individual CCFs were combined at the end of the analysis. For computational reasons, we resampled all of the spectra onto a common wavelength grid of $0.01 \AA$ spacing. We identified bad pixels (e.g., due to cosmic-ray hits) by selecting $5 \sigma$ outliers in a sliding 500 pixel window, and setting them to the mean of the values in the window. We shifted each spectrum into the rest frame of the star, taking the $-21.07 \mathrm{~km} \mathrm{~s}^{-1}$ systemic velocity (denoted $V_{\text {sys }}$ ) (Talens et al. 2018) and the barycentric Earth RV (BERV) correction into account (calculated using the Astropy package, see Astropy Collaboration 2013, 2018). The BERV changed by $\lesssim 1 \mathrm{~km} \mathrm{~s}^{-1}$ over the course of the transit, and we did not correct for stellar reflex motion. The latter effect is negligible because M2 has broad absorption lines due to its fast rotation. We note the presence of excess absorption in the cores of the $\mathrm{Na}$ doublet, which we attribute to stationary interstellar medium (ISM) absorption, as seen in previous analyses of M2 (Casasayas-Barris et al. 2018, 2019). As in these studies, neglecting the stellar reflex correction helps ensure that the ISM absorption cancels during division by the master out-of-transit spectrum (see below). The $2.5 \%$ of pixels on either end of each order were masked from the cross-correlation. This step prevented edge artifacts from shifting to the stellar rest frame, as well as pixels with low flux located at the edges of the blaze function. 
We coadded all out-of-transit spectra $\left\{f_{t}\right\}_{t \in t_{\text {out }}}$ to obtain a master spectrum of the star $F_{\text {out }}$. We subsequently computed individual transmission spectra $\left\{\widetilde{r}_{t}\right\}_{t \in t_{\text {in }}}$ by dividing each intransit spectrum $\left\{f_{t}\right\}_{t \in t_{\text {in }}}$ by $F_{\text {out }}$ (Wyttenbach et al. 2015; Allart et al. 2017; Hoeijmakers et al. 2018a; Casasayas-Barris et al. 2018, 2019). Finally, we applied a high-pass Gaussian filter with 75 pixel standard-deviation and subtracted from the pixels in each wavelength bin their time average, removing broadband variations (Hoeijmakers et al. 2018b).

\subsection{Cross-correlation}

Atomic or molecular species present in the exoplanet atmosphere may cause thousands of individual absorption lines in the atmospheric transmission spectrum. Cross-correlating the spectra with a model template spectrum combines the contributions of all these lines to reduce the photon-noise and yields significant detections of these elements (Snellen et al. 2010). We used high-resolution model spectra for $\mathrm{Na} \mathrm{I,} \mathrm{Mg} \mathrm{I,} \mathrm{Sc} \mathrm{I,} \mathrm{Sc} \mathrm{II,} \mathrm{Ti} \mathrm{I,} \mathrm{Ti} \mathrm{II,}$ CrI, CrII, Fe I, Fe II, and Y II using opacities computed with HELIOS-K (Grimm \& Heng 2015) and equilibrium chemistry at $T=4000 \mathrm{~K}$ with FastChem (Stock et al. 2018). These templates resulted in detections in the analysis of the transmission spectrum of KELT-9 b by Hoeijmakers et al. (2019), who provided a detailed description of their construction. These templates are publicly accessible via the $\operatorname{CDS}^{11}$.

To compute the cross-correlation, we applied Doppler shifts of -500 to $500 \mathrm{~km} \mathrm{~s}^{-1}$ to the template in increments of $1.0 \mathrm{~km} \mathrm{~s}^{-1}$. At each velocity $v$, and for a given transmission spectrum $\widetilde{r}_{t}$, we took the weighted average of values in the spectrum. The weights are a product of the Doppler-shifted template, which is zero everywhere except at locations of line transitions, and the inverse of the time variance of each wavelength bin, which reduces the contribution of noisy pixels (Brogi et al. 2016). This procedure yields a cross-correlation function $(\mathrm{CCF})$ as a function of velocity and time that contains the average line strength of features in the transmission spectrum (Hoeijmakers et al. 2019).

\subsection{Rossiter-McLaughlin effect}

The orbit of M2 b is aligned (Lund et al. 2017; Talens et al. 2018), meaning that the planet obscures the blueshifted part of the star at the start of the transit, and moves to the redshifted part toward egress. This passage across the stellar disk changes the shapes of the disk-integrated rotation-broadened stellar absorption lines, which gives rise to the RM effect seen in the RV measurements of the star during transit (Ohta et al. 2005). For a fast rotator like $\mathrm{M} 2$, broadened spectral features decrease the precision of RV measurements, making the RM effect difficult to resolve. However, when the mean out-of-transit spectrum is divided by each of the exposures, the line deformation causes the stellar lines to be overcorrected at the instantaneous velocity of the obscured part of the stellar disk. This effect is known as the "Doppler shadow", which is cast by the planet as it progresses through transit (Collier Cameron et al. 2010). The centroid velocity $v_{*}(t)$ of the obscured stellar surface depends on the projected location of the planet with respect to the stellar spin axis, which changes over time as the planet progresses through transit, and is described by the following analytical expressions (Collier Cameron et al. 2010; Bourrier et al. 2015), ignoring differential rotation of the stellar surface and convective blueshift (Cegla et al. 2016):

$v_{*}(t)=x_{\perp}(t) v_{\mathrm{eq}} \sin i_{*}$,

11 https://cdsarc.unistra.fr/viz-bin/cat?]/A+A/627/A165 where $v_{\text {eq }} \sin i_{*}$ is the projected equatorial rotation velocity of the star, and $x_{\perp}$ is the orthogonal distance of the obscured region to the projected spin axis,

$x_{\perp}(t)=x_{p}(t) \cos (\lambda)-y_{p}(t) \sin (\lambda)$,

where $\lambda$ is the spin-orbit misalignment, and $x_{p}(t)$ and $y_{p}(t)$ are the coordinates of the planet,

$x_{p}(t)=\frac{a}{R_{*}} \sin (2 \pi \phi)$
$y_{p}(t)=-\frac{a}{R_{*}} \cos (2 \pi \phi) \cos \left(i_{p}\right)$,

with $a$ the semimajor axis, $R_{*}$ the radius of the star, $i_{p}$ the orbital inclination, $\phi$ the orbital phase at time $t$, and the orbital inclination $i_{p}$.

The Doppler shadow was retrieved by cross-correlating the transmission spectra $\left\{\widetilde{r}_{t}\right\}_{t \in t_{\text {in }}}$ with a model template of the stellar spectrum. This template consisted of a PHOENIX photosphere model spectrum at a temperature of $9000 \mathrm{~K}$ and solar metallicity, obtained from the online PHOENIX library (Husser et al. 2013). The baseline of the template was subtracted with a high-pass filter so that it contained only the absorption lines, with relative depths corresponding to the relative absorption of continuum radiation at each wavelength (i.e., continuum normalization).

The resulting 2D CCF contains the signature of the Doppler shadow (see the top panel in Fig. 2), from which the misalignment $\lambda$ between the orbital plane of the planet and the projected stellar spin axis can be derived, as is done when Doppler tomography (Collier Cameron et al. 2010) or the so-called reloaded RM effect is applied (Cegla et al. 2016). The shadow also overlaps with the expected RV of the planet, therefore it needs to be corrected before the signature of the planet atmosphere may be isolated (see the second panel, RM RV model, in Fig. 2; the local obstructed RV overlaps the planetary RV, given by dashed and dotted lines, respectively). We constructed a model of the 2D cross-correlation residual from the parameters of the Gaussian fits used to measure $\lambda$, following the approach by Hoeijmakers et al. (2018a). This model was scaled to minimize the sum of the squared residual when subtracting it from CCFs with other the templates used in this analysis (see the third and fourth panels, shadow model and residual, in Fig. 2). The Doppler shadow shows small variations in strength throughout the transit; darker regions are roughly correlated with higher exposure $\mathrm{S} / \mathrm{N}$. However, our empirical model is agnostic to the origin of these variations, and successfully removed the shadow to isolate the atmospheric absorption. The overlap region between the atmospheric absorption trail and the Doppler shadow may affect the coadded absorption signal; however, as described by Hoeijmakers et al. (2018a), the fit of the Doppler shadow model ignores the overlap region and enforces smoothly varying model parameters, which helps to preserve information at velocities for which the Doppler shadow and the planet absorption features overlap. This is possible because the range of RVs spanned by the Doppler shadow differs significantly from the ranges of the planet atmosphere because the host star rotates so fast.

\subsection{Time-averaged CCF}

The apparent RV of the planet is described by

$v(t)=K_{\mathrm{p}} \sin (2 \pi \phi(t))+V_{\mathrm{sys}}$,

where $K_{\mathrm{p}}$ is the planetary semiamplitude. Because we shifted all spectra to the stellar rest frame, the $V_{\text {sys }}$ term above is set 


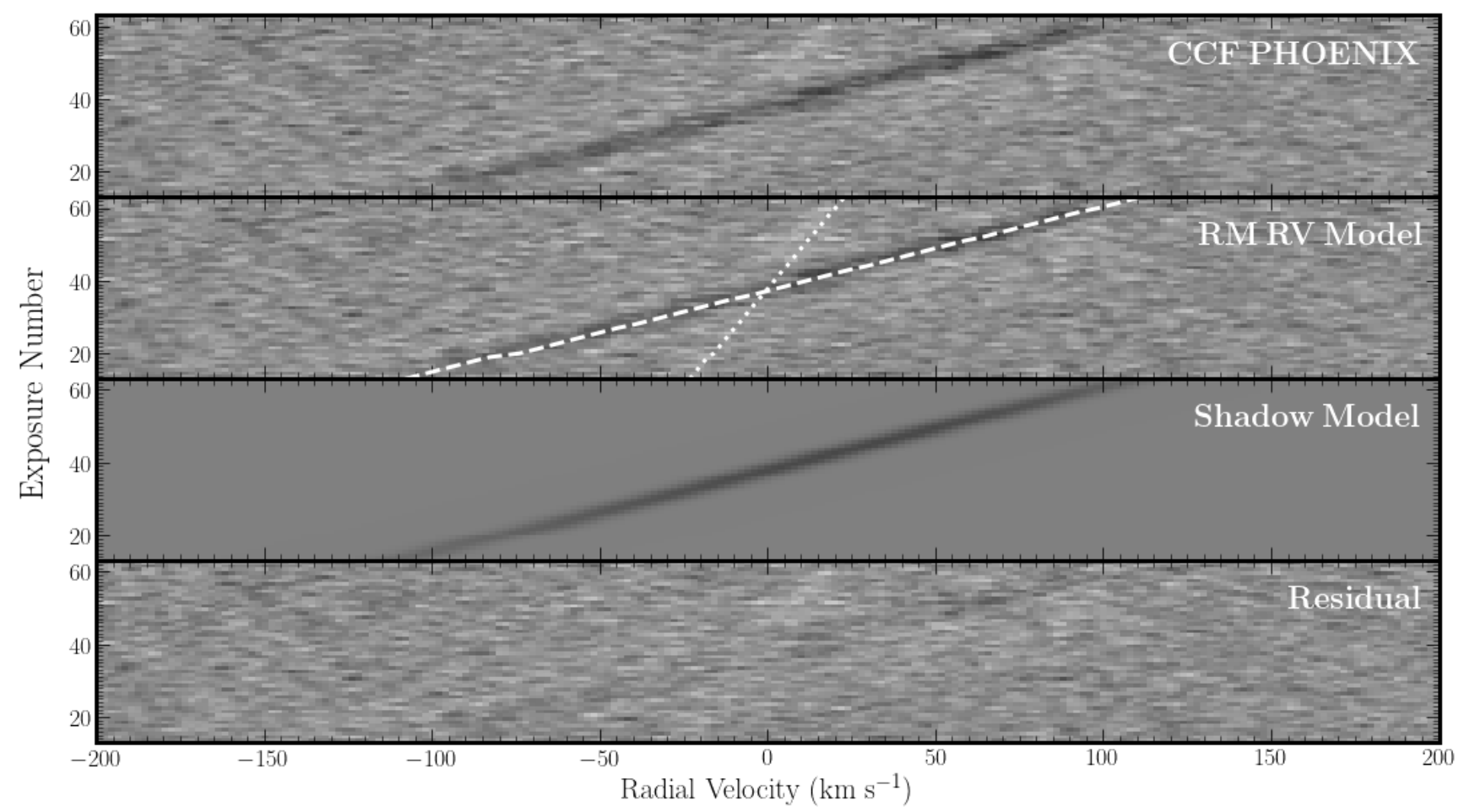

Fig. 2. Removal of the Doppler shadow caused by the transiting planet from the start of ingress to the end of egress. Top panel: cross-correlation of the exposures in-transit with a template constructed from a PHOENIX stellar photosphere model. Second panel: same as the top panel, annotated with the predicted RV of the occulted stellar region responsible for the Doppler shadow (dashed line). The expected RV of the planet (dotted line) expected from the system parameters by Lund et al. (2017) and Talens et al. (2018) is also shown. Third panel: model of the Doppler shadow obtained by fitting a Gaussian profile to the shadow feature in each of the in-transit CCFs. Bottom panel: residuals after subtraction.

to $0.0 \mathrm{~km} \mathrm{~s}^{-1}$. The orbital period $P=3.474119$ days and reference mid-transit time $T_{0}=57909.0875$ MJD (Talens et al. 2018) determine the orbital phase, $\phi(t)$ at the time of each exposure. We sample potential values for $K_{\mathrm{p}}$ from a grid ranging from $0-300 \mathrm{~km} \mathrm{~s}^{-1}$ in steps of $1.0 \mathrm{~km} \mathrm{~s}^{-1}$ and shift each CCF by $-K_{\mathrm{p}} \sin (2 \pi \phi(t))$. At the true value of $K_{\mathrm{p}}$, the CCFs are shifted into the rest frame of the planet, and may be optimally coadded to yield the time average of the CCFs as a function of the systemic velocity $V_{\text {sys. }}$. The planet signal is expected to occur at $0.0 \mathrm{~km} \mathrm{~s}^{-1}$ because the spectra have been shifted to the stellar rest frame. In this way, we construct a $2 \mathrm{D}$ map of the coadded cross-correlation signal at different combinations of $K_{\mathrm{p}}$ and $V_{\text {sys }}$, which has been termed the $K_{p} V_{\text {sys }}$ diagram (Brogi et al. 2012). A statistically significant signal at the correct combination of $K_{p}$ and $V_{\text {sys }}$ confirms the presence of the model species in the atmosphere of the planet (Brogi et al. 2012). Additionally, we can examine a single row in this map that corresponds to any particular choice of $K_{\mathrm{p}}$. The peak in this coadded CCF corresponds to the weighted mean of the depths of the absorption lines in the chosen rest frame (Pino et al. 2018; Hoeijmakers et al. 2019). The orbital period and semimajor axis of M2 (Talens et al. 2018) and previous atmospheric measurements (Casasayas-Barris et al. 2019; Nugroho et al. 2020; Stangret et al. 2020) place $K_{\mathrm{p}}$ roughly between 160 and $190 \mathrm{~km} \mathrm{~s}^{-1}$.

\section{Results}

\subsection{Spin-orbit misalignment}

By making use of the fact that the RV variation of the Doppler shadow traces the obscured area of the stellar disk as the planet moves through transit, the system architecture can be derived (Collier Cameron et al. 2010). To this end, we normalize the 2D CCF by the expected flux decrease during the planet transit (i.e., the transit light curve) and fit the centroid position of the correlation excess with a Gaussian profile in the CCF of each exposure, following Cegla et al. (2016) (see Fig. 3). We take the centroid $v_{*}^{\mathrm{obs}}(t)$ and its uncertainty $\sigma_{v}^{\mathrm{obs}}(t)$ as our measurement of the average RV of the occulted stellar surface. That is, $v_{*}^{\text {obs }}(t)$ represents the measurement of $v_{*}(t)$ that is defined in Eq. (1). We assume a standard Gaussian log-likelihood function,

$\mathcal{L} \propto-\frac{1}{2} \sum_{t}\left(\frac{v_{*}^{\mathrm{obs}}(t)-\hat{v}_{*}(t)}{\sigma_{v}^{\mathrm{obs}}(t)}\right)^{2}$,

where $\hat{v}_{*}(t)$ is the model-predicted value of $v_{*}(t)$. We subsequently perform a Markov chain Monte Carlo (MCMC) analysis of the Doppler shadow to constrain the orbital parameters of the system.

We assumed Gaussian priors on each of the orbital parameters, with mean and standard deviation as reported by Talens et al. (2018) (see Table A.1). Our model contained five parameters: scaled semimajor axis $a / R_{*}$, projected obliquity $\lambda$, orbital inclination $i_{p}$, projected stellar rotation speed $v \sin i_{*}$, and a systematic RV offset to account for errors on the systemic velocity. We placed a Gaussian prior on the offset with mean and standard deviation of 0.0 and $5.0 \mathrm{~km} \mathrm{~s}^{-1}$, respectively. The MCMC was performed with the emcee package (Foreman-Mackey et al. 2013), using 20 independent chains, each taking $1 \times 10^{5}$ steps. Visual inspection of the chains suggested a burn-in time of $\sim 200$ steps, and an auto-correlation length of about steps. We excluded the first 5000 steps of each chain and nine out of every ten 

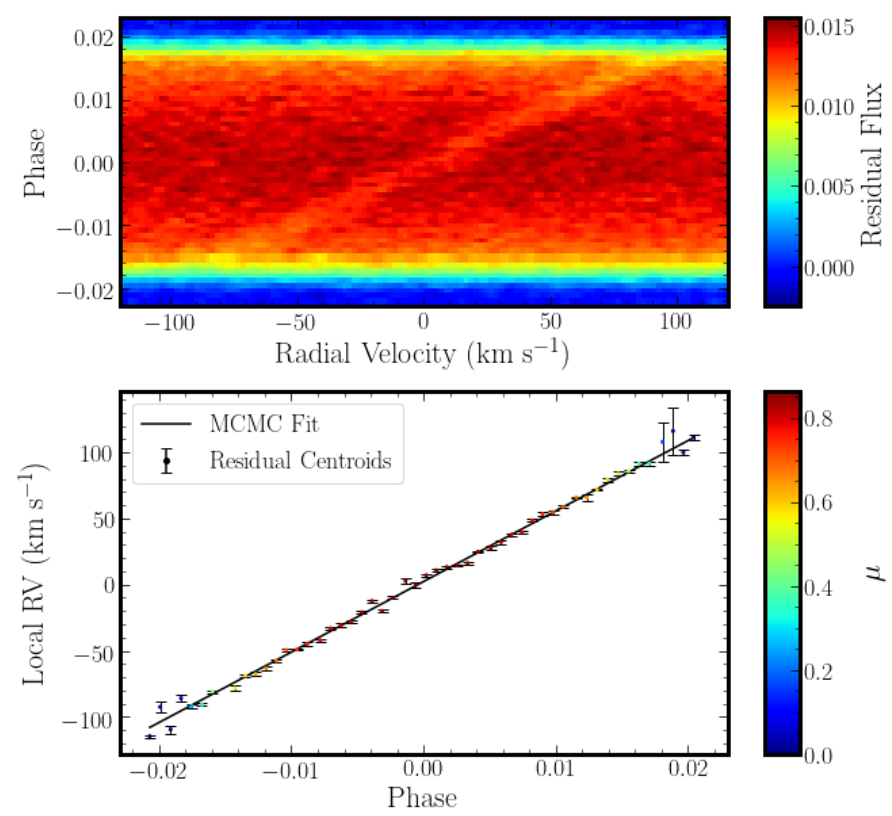

Fig. 3. Top panel: two-dimensional CCF obtained using the PHOENIX stellar template, normalized by the transit light curve. Bottom panel: functional form of the time-dependent centroid velocity of the Doppler shadow is fit using an MCMC optimizer, providing posterior distributions of the system parameters (see Fig. A.1).

remaining steps, and subsequently merged the chains, leaving $>10^{5}$ independent samples of the posterior. Correlation diagrams and histograms of each parameter are shown in Fig. A.1, and the median values are quoted in Table A.1. Our results are generally consistent with those reported by Talens et al. (2018) and Lund et al. (2017). The marginalized distributions reduce the $1 \sigma$ uncertainty in $v \sin i_{*}$ to $<1 \mathrm{~km} \mathrm{~s}^{-1}$, slightly improve constraints on $\lambda$, and constrain an absolute RV offset to within a few $\mathrm{km} \mathrm{s}^{-1}$. Distributions for $a / R_{*}$ and $i_{p}$ are dominated by the choice of priors, which are provided by the already tight constraints derived from transit photometry (Talens et al. 2018). Our best-fit parameters, in combination with Eqs. (1)-(4), and the orbital phase of each exposure, trace the path of the Doppler shadow (second panel, RM RV model, Fig. 2). Importantly, we note a degeneracy between RV offset and $\lambda$, which highlights the need for precise measurements of $V_{\text {sys }}$ when fitting for the spinorbit misalignment. However, it is difficult to accurately measure $V_{\text {sys }}$ for fast rotators such as M2, and literature estimates vary by several $\mathrm{km} \mathrm{s}^{-1}$.

\subsection{Transmission spectrum and cross-correlation}

We present new detections of $\mathrm{Cr}$ II $(4.1 \sigma)$ and $\mathrm{Mg}$ I $(4.0 \sigma)$ in the atmosphere of $\mathrm{M} 2 \mathrm{~b}$, in addition to confirmations of Fe I $(4.7 \sigma)$, Fe II $(4.8 \sigma)$, and Na I $(4.4 \sigma)$ (Casasayas-Barris et al. 2019; Nugroho et al. 2020; Stangret et al. 2020). The crosscorrelation procedure and detection of Fe II is shown in Fig. 4; the same plots for the remaining species are presented in the appendix.

Several metrics have previously been used to determine detection significance in CCFs, including the amplitude of the $\mathrm{CCF}$ peak relative to the standard deviation of noise in the baseline of the CCF (Brogi et al. 2012), a Welch t-test comparing distributions in the 2D CCF (Birkby et al. 2017), and the uncertainty in a Gaussian fit to the CCF feature (Hoeijmakers et al.
2019). The significances determined in the present work represent the false-alarm probability (FAP) of detecting a comparable CCF enhancement by chance. We discuss the FAP calculation in the following section. Summary statistics for each detected species, including the CCF peak, Gaussian fit, and FAP, are listed in Table 1.

Our best-fit relative depth of Fe II $(0.013 \pm 0.002 \%)$ is consistent with the $0.08 \pm 0.04 \%$ depth of individual lines reported in combined HARPS-N data (Casasayas-Barris et al. 2019) within $\lesssim 2 \sigma$. Likewise, our Na I depth $(0.139 \pm 0.015 \%)$ is comparable to the previously reported $0.09 \pm 0.05 \%$ combined strength of the Na D1 and D2 lines. In addition, all detected species have depths comparable to those found in the atmosphere of the ultrahot Jupiter KELT-9 b (Hoeijmakers et al. 2019), but in contrast with KELT-9 b, this transit observation provides no evidence of absorption of Ti II, Sc II, or Y II. We fixed $K_{p}$ at $175 \mathrm{~km} \mathrm{~s}^{-1}$, consistent with the various optimal values of $K_{p}$ found by Casasayas-Barris et al. (2019), Nugroho et al. (2020), and Stangret et al. (2020). Variations of several $\mathrm{km} \mathrm{s}^{-1}$ in $K_{p}$ do not change the recovered signal amplitudes or confidence levels appreciably.

To explain this discrepancy, we note that there is a difference in equilibrium temperature of almost $2000 \mathrm{~K}$ between M2 $\left(T_{\text {eq }} \sim 2260 \mathrm{~K}\right)$ and the much hotter KELT-9 b $\left(T_{\text {eq }} \sim 4050 \mathrm{~K}\right.$ Gaudi et al. 2017), therefore it is plausible that significant differences exist between the atmosphere chemistry and thermal structure of the atmospheres of these two planets. In addition, the present observations were obtained over the course of a single transit, whereas two transits of KELT-9 b were used by Hoeijmakers et al. (2019). As a result, the combined CCFs have a lower $\mathrm{S} / \mathrm{N}$ than what was achieved in the analysis of KELT-9 $\mathrm{b}$, resulting in lower sensitivities to trace species such as Sc II or Y II, which were also least strongly detected in the sample of Hoeijmakers et al. (2019).

With regard to the nondetection of Ti II, which was strongly detected in KELT-9 b, it is worth noting that the equilibrium temperature of M2 is slightly below that of WASP-121 b ( $T_{\text {eq }} \sim$ $2358 \mathrm{~K}$ ), for which $\mathrm{TiO}$ condensation has been observed to be important (Delrez et al. 2016; Evans et al. 2018). The peak locations of the CCF features generally suggest a blueshift of $\sim-1$ to $-9 \mathrm{~km} \mathrm{~s}^{-1}$, which may be indicative of a day- to nightside wind or systematic errors in the determination of the systemic velocity, which can be difficult to constrain for fast rotators (Hoeijmakers et al. 2019). However, Nugroho et al. (2020) and Stangret et al. (2020) recently also report blueshifts of the Fe I and Fe II signals of a few $\mathrm{km} \mathrm{s}^{-1}$, with Fe II generally showing a lower blueshift than Fe II. The interpretation that atmospheric absorption lines are significantly blueshifted therefore appears to be robust across multiple independent studies, indicating the presence of a day- to nightside wind. This wind appears to affect Fe I and Fe II differently, suggesting a stratification of the atmosphere, as has also been hypothesized by Nugroho et al. (2020).

The CCF for TiII exhibits a slight enhancement at $0.0 \mathrm{~km} \mathrm{~s}^{-1}$ with an $S / N \sim 2.4$. While this peak is the highest in the $1 \mathrm{D} \mathrm{CCF}$, we refrain from claiming a detection. All detections listed in Table 1 have an $\mathrm{S} / \mathrm{N}$ of at least 3.0 and correspond to the maximum in the $1 \mathrm{D} \mathrm{CCF}$. These two criteria were not met by the CCF of any other species.

The absorption signature of $\mathrm{MgI}$ appears significantly broader than that of the other species, with a measured FWHM of $33.45 \pm 3.30 \mathrm{~km} \mathrm{~s}^{-1}$ vs. $\sim 10 \mathrm{~km} \mathrm{~s}^{-1}$ for the other species. On their own, the current data do not provide evidence for identifying the cause of broadening processes that would specifically act on $\mathrm{Mg}$ I. However, we note that diverse 

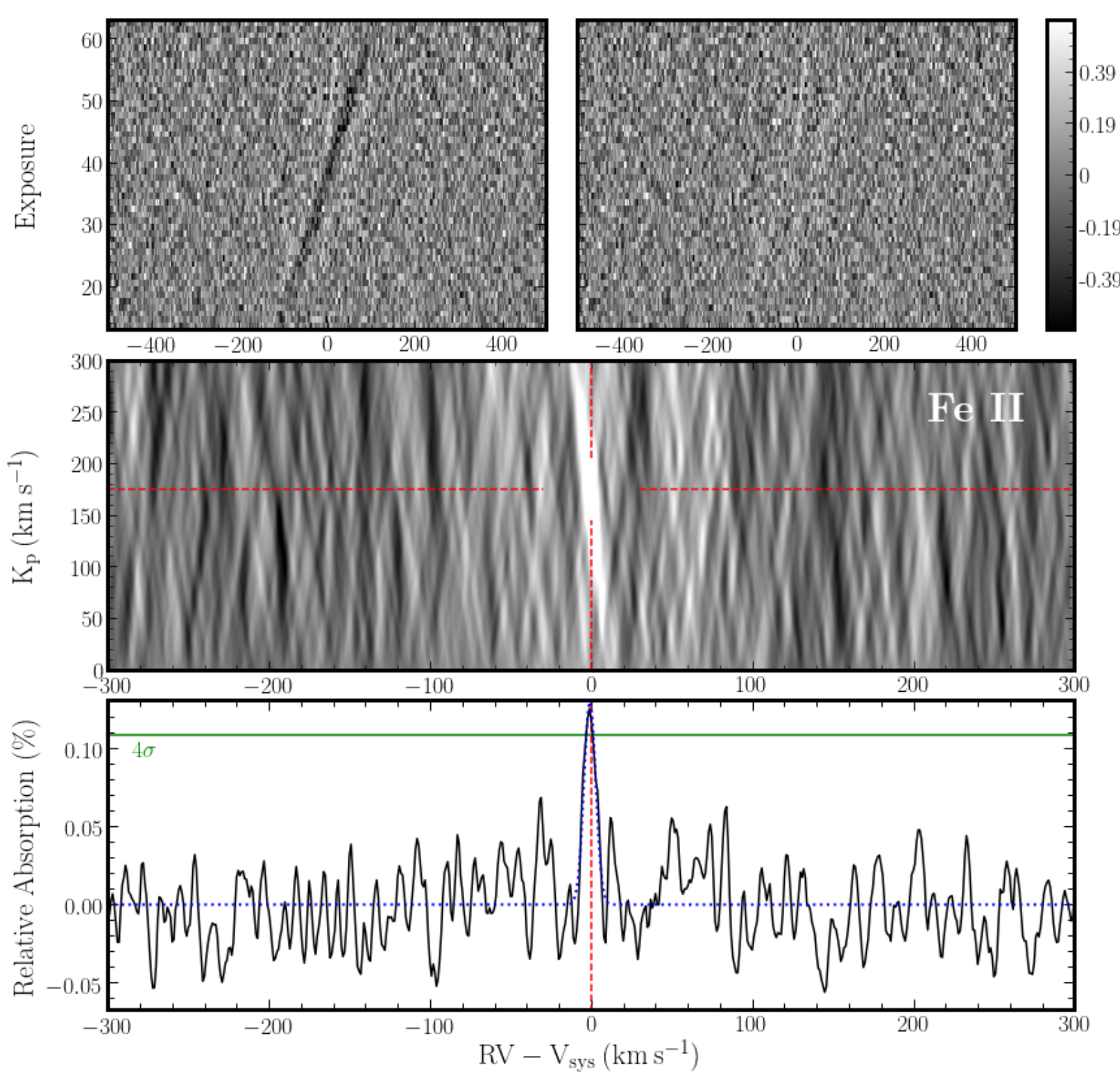

Fig. 4. Cross-correlation procedure and detection of Fe II in the transmission spectrum of MASCARA-2 b. Upper panels: two-dimensional crosscorrelation with the Fe II template before (left) and after (right) correction for the Doppler shadow. Middle panel: $K_{p} V_{\mathrm{sys}}$ diagram. The dashed red lines indicate the expected location of the atmospheric signal. Because the data are shifted by the systemic velocity and BERV, the signal lies at approximately 0 along the $x$-axis. Bottom panel: CCF coadded in the rest frame of the planet. The green line marks four times the standard deviation of the CCF. An enhancement is detected at the rest-frame velocity of the planet, which is modeled with a Gaussian profile (dotted blue line).

Table 1. Summary of detected atomic species.

\begin{tabular}{|c|c|c|c|c|c|c|c|c|}
\hline Species & $\mathrm{CCF}_{\max }(\%)$ & $S / N$ & $v_{\mathrm{CCF}}\left(\mathrm{km} \mathrm{s}^{-1}\right)$ & $A(\%)$ & $\mu\left(\mathrm{km} \mathrm{s}^{-1}\right)$ & $F W H M\left(\mathrm{~km} \mathrm{~s}^{-1}\right)$ & $p_{\text {FAP }}$ & $\sigma_{\text {FAP }}$ \\
\hline $\mathrm{Fe} I$ & 0.015 & 3.45 & -2 & $0.013 \pm 0.002$ & $-4.81 \pm 0.72$ & $12.39 \pm 1.69$ & $1.19 \mathrm{e}-06$ & 4.72 \\
\hline $\mathrm{Fe}$ II & 0.125 & 4.60 & -1 & $0.130 \pm 0.011$ & $-0.75 \pm 0.37$ & $8.54 \pm 0.87$ & $9.72 \mathrm{e}-07$ & 4.76 \\
\hline Cr II & 0.120 & 3.69 & -3 & $0.117 \pm 0.019$ & $-3.40 \pm 0.42$ & $5.31 \pm 0.99$ & $2.47 e-05$ & 4.06 \\
\hline $\mathrm{NaI}$ & 0.126 & 3.40 & -2 & $0.139 \pm 0.015$ & $-4.38 \pm 0.54$ & $10.07 \pm 1.26$ & $3.96 \mathrm{e}-06$ & 4.47 \\
\hline Mg I & 0.084 & 3.33 & -3 & $0.062 \pm 0.005$ & $-8.40 \pm 1.40$ & $33.45 \pm 3.30$ & $3.51 \mathrm{e}-05$ & 3.98 \\
\hline
\end{tabular}

Notes. Columns 1-4: species name, peak relative absorption, S/N, and location of the peak in the 1D CCF. Columns 5-7: amplitude, centroid, and FWHM of a Gaussian profile fit to the CCF feature, along with corresponding uncertainties. Columns 8 and 9: false-alarm-probabilities and confidence level assuming Gaussianity. 
broadening has also been observed in the transmission spectrum of KELT-9 b, with $\mathrm{Na}$ I and Mg I showing FHWMs of 27.8 \pm 3.7 and 27.5 \pm 4.3 , respectively (Hoeijmakers et al. 2019), as opposed to values between 10 and $20 \mathrm{~km} \mathrm{~s}^{-1}$ for other species. Assuming that line broadening is mainly due to atmospheric dynamics, differences in the line widths of different species would suggest that certain species may exist in distinct dynamical regimes in the upper atmospheres of ultrahot Jupiters. This could include day- to nightside flows versus super-rotational jets (Showman et al. 2013; Louden \& Wheatley 2015; Brogi et al. 2016) or radial outflows (Seidel et al. 2019). However, further analysis and observations are needed to clarify the mechanism by which the absorption lines of certain species may be differentially broadened by these effects, and to what extent these processes are common among ultrahot Jupiters.

\section{Discussion and conclusion}

\subsection{Bootstrap and temporal variation}

The statistical treatment of the signatures as quoted above assumes that the noise in the CCF is normally distributed and uncorrelated. To determine the robustness of the detected signals, we determined FAPs using a bootstrap approach as follows. For a given species, we started with the 2D CCF time series after removing the Doppler shadow. We randomly shuffled each row of the CCF, coadded in the rest frame of the planet, fitted a Gaussian profile at $0.0 \mathrm{~km} \mathrm{~s}^{-1}$ (i.e., the center of the randomly shuffled rows), and recorded the fitted amplitude. This process was repeated 50000 times to populate a random distribution of amplitudes. When fitting the Gaussian, we enforced an $F W H M \geq$ $5 \mathrm{~km} \mathrm{~s}^{-1}$ and a centroid $|\mu| \leq 20 \mathrm{~km} \mathrm{~s}^{-1}$. By setting a minimum FWHM, we required that a spurious CCF enhancement must be sufficiently wide to qualify as a false alarm. Narrower absorption line profiles would be inconsistent with the minimum width set by the rotation of the planet, assuming tidal locking. All of the reported detections in Table 1 have $F W H M>5 \mathrm{~km} \mathrm{~s}^{-1}$. Finally, we fitted the tail of the amplitude distribution with a power-law model and extrapolated it to the strength of the detected signal. When we assume that the distribution is Gaussian, the FAP can be converted into a $\sigma$-confidence level. The distribution of random amplitudes is shown in Fig. 5 for the case of Fe II. Distributions for other species are presented in the appendix.

The FAP does not account for the autocorrelation between absorption lines from a given species or for spurious correlations between lines from different species. Because several CCFs show strong spurious signals (offset from the $0.0 \mathrm{~km} \mathrm{~s}^{-1}$ mark), we manually confirm the autocorrelation function of each model template. Additionally, we verified the CCF between each template and Fe I and Fe II because these species contribute a multitude of strong absorption lines. The peak in the Mg I CCF at $+80 \mathrm{~km} \mathrm{~s}^{-1}$ is due to spurious correlation with a strong nearby Fe II line that was also observed by Hoeijmakers et al. (2019). Fe II also produces a signal with Cr II near $+50 \mathrm{~km} \mathrm{~s}^{-1}$. Autocorrelations for the detected species do not produce significant spurious signals, and spurious correlations with $\mathrm{Fe}$ I are at much lower amplitude than the detections. Spurious features in CCFs of Na I near $+90 \mathrm{~km} \mathrm{~s}^{-1}$, Cr II near $+20 \mathrm{~km} \mathrm{~s}^{-1}$, and Ti II near $+90 \mathrm{~km} \mathrm{~s}^{-1}$ and $+170 \mathrm{~km} \mathrm{~s}^{-1}$ cannot be easily explained, and may result from noise in the $2 \mathrm{D} \mathrm{CCF}$.

The measured planetary absorption signal appears to be stronger in the second half of the transit than it does in the first half. This is most evident in the upper right panel of Fig. 4, where
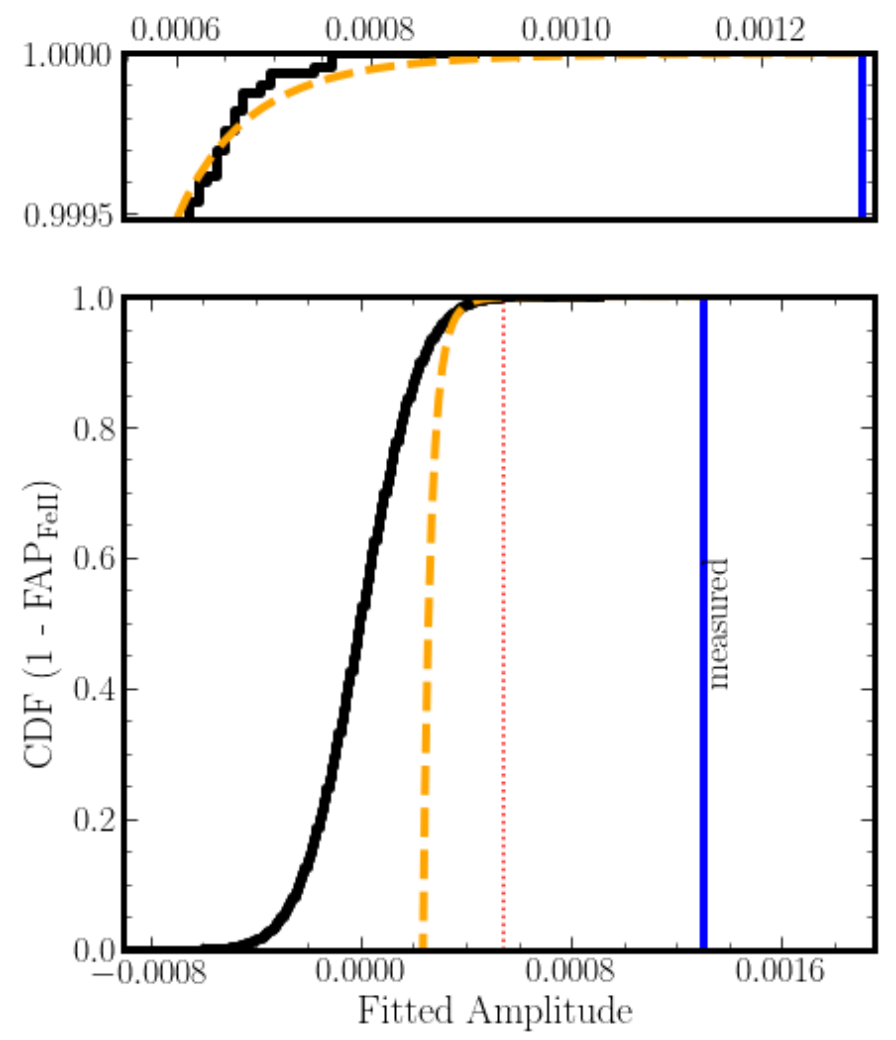

Fig. 5. Cumulative distribution function of amplitudes of Gaussian fits to the randomly shuffled and stacked Fe II CCF. The black curve depicts the CDF. The solid blue line marks the strength of the Fe II detection. The dotted red line marks three standard deviations from the distribution mean, after which the distribution is fitted with a power law, shown as a the dashed orange line. The upper panel zooms in on the tail of the CDF for clarity.

the signature of the Fe II line appears visible by eye during the later exposures of the observing sequence. While this could be stochastic, particularly because this analysis is based on a single transit, it may instead be a physical effect related to the distribution of Fe II in the atmosphere of the planet, where it is present on the dayside near the evening (trailing) terminator that rotates into view toward the end of the transit. This effect has recently been used to explain time-dependencies in the absorption spectrum of Fe I in the atmospheres of WASP-121 b (Bourrier et al. 2020) and WASP-76 b (Ehrenreich et al. 2020). We quantified this trend by fitting a Gaussian profile to the $1 \mathrm{D}$ coadded $\mathrm{CCF}$ feature using only the first and second halves of the in-transit exposures separately (Fig. 6). We took the difference between the two Gaussian amplitudes of Fe II (which shows the largest relative discrepancy between transit halves) and compared this to differences that might arise by chance, given a signal that is uniform throughout the transit.

First, we added a Gaussian absorption line of identical amplitude and FWHM as the detected absorption line to each row of the 2D CCF, thereby injecting an artificial signal of identical strength. The centroid was set by the corresponding phase, and a randomly selected $0<K_{p}<300 \mathrm{~km} \mathrm{~s}^{-1}$, and $-300<V_{\text {sys }}<$ $300 \mathrm{~km} \mathrm{~s}^{-1}$ (excluding $-20<V_{\text {sys }}<20 \mathrm{~km} \mathrm{~s}^{-1}$ to avoid overlap with the actual planet signal). We subsequently stacked the 2D $\mathrm{CCF}$ along the velocities of the injected signal, treating the first and second halves separately. We fitted new Gaussians at the $V_{\text {sys }}$ of the injected signal for each half, and recorded the difference in their amplitudes. We repeated this procedure 10000 times, 

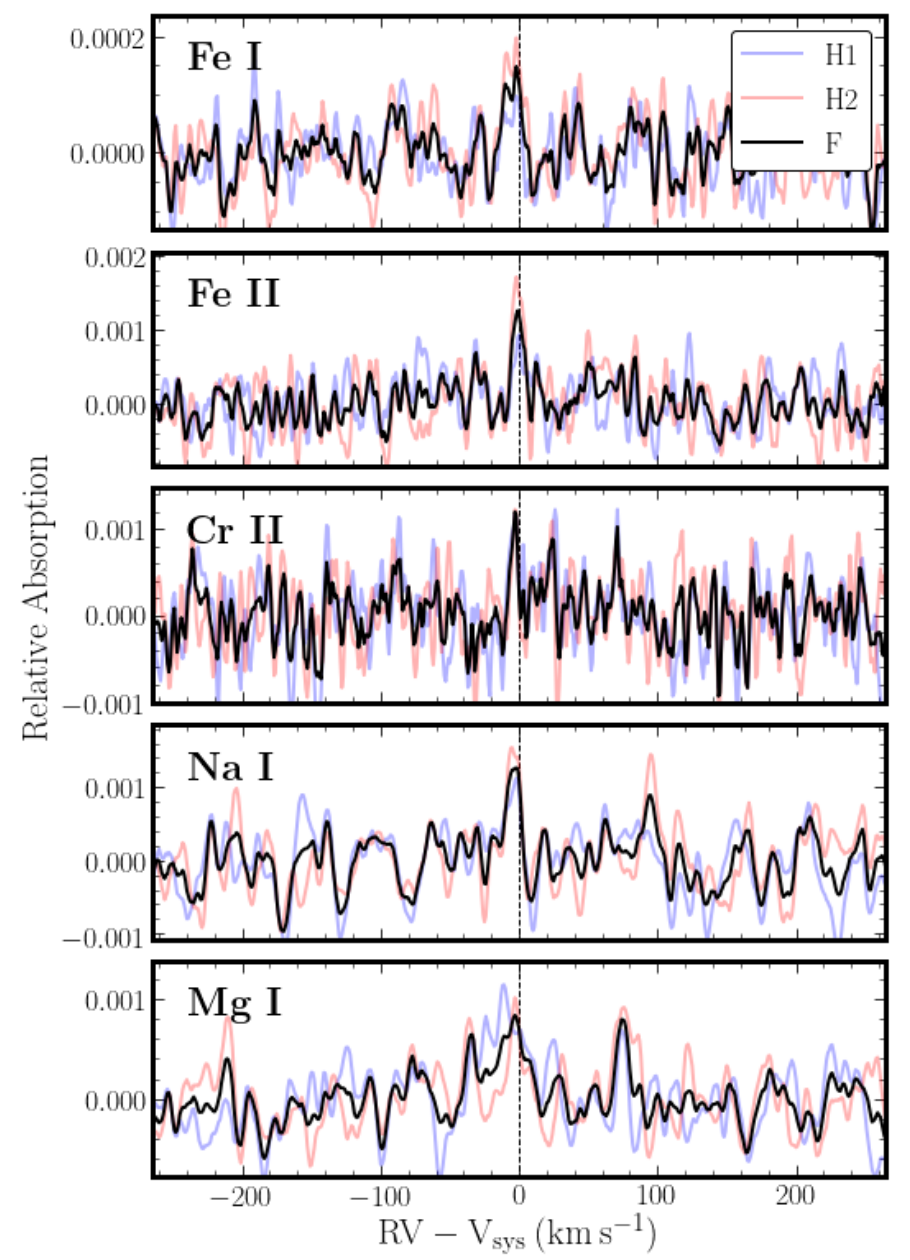

Fig. 6. Comparison of coadded CCF features using data from the first half of transit (blue curve), the second half (red curve), and the full transit (black curve) for each of the strongest species detections (Fe I, $\mathrm{Fe}$ II, and $\mathrm{Cr}$ II). The legend denotes which in-transit exposures were used in the calculation $(\mathrm{H} 1, \mathrm{H} 2$, and $\mathrm{F}$ denote the first half of transit, the second half of transit, and the full transit, respectively). The dashed line indicates the expected location of the signal, at $0.0 \mathrm{~km} \mathrm{~s}^{-1}$ velocity offset.

injecting and recovering a signal along random paths in the 2D CCF. The results are shown in Fig. 7, where the observed difference or greater occurs in $1.2 \times 10^{-3}$ of all cases. When we assume that the distribution is Gaussian, the observed Fe II amplitude difference is at a $2.7 \sigma$ confidence level.

Variability of the depth of absorption features has been observed in the transmission spectra of KELT-9 b (Cauley et al. 2019) and currently most clearly in WASP-76 b (Ehrenreich et al. 2020). In the case of WASP-76 b, this is attributed to differences in chemical composition between the morning (leading) and evening (trailing) terminators, due to the offset of the substellar hot spot toward the evening terminator. These observations show the appearance of a significant blueshift as the evening twilight region rotates into view, which is explained by the rotation of the planet in combination with a day- to nightside wind: the transmission spectrum of the leading terminator probes cool gas that streams from the nightside toward the hot dayside. Conversely, the trailing limb contains hot gas that was strongly irradiated on the dayside and is approaching the nightside, where it cools down. However, in the case of M2 b, the absorption signal appears to be symmetric around $0 \mathrm{~km} \mathrm{~s}^{-1}$, which may indicate

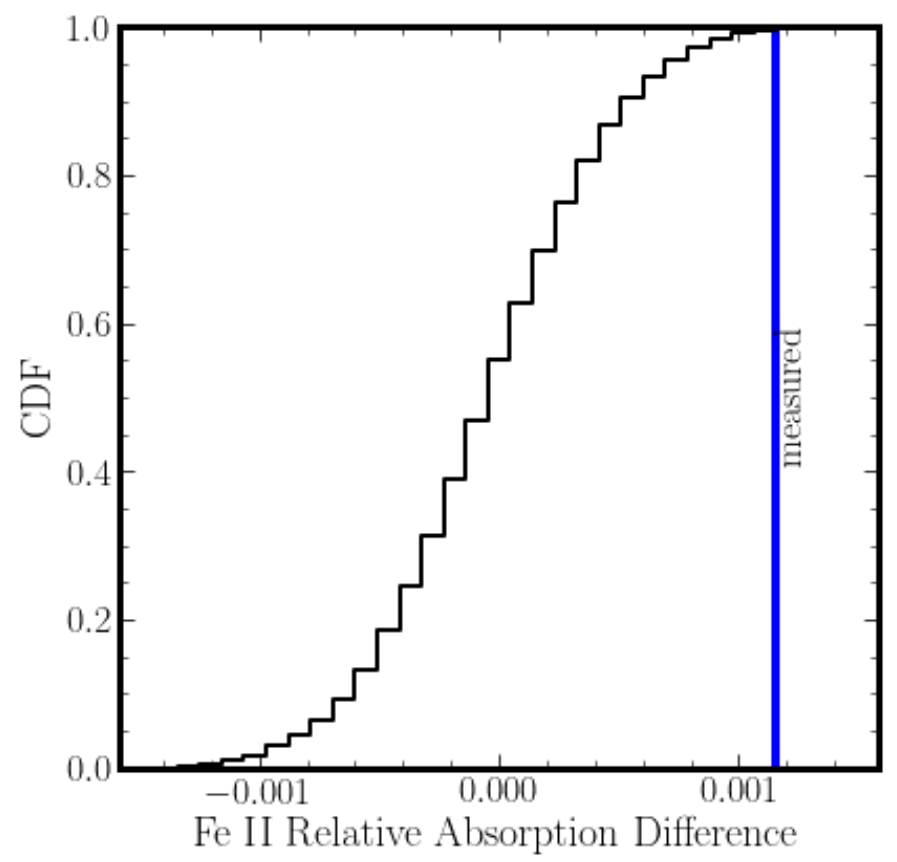

Fig. 7. Cumulative distribution function of the difference in amplitudes recovered from injecting an artificial Fe II signal, and recovering it in the first and second halves of transit separately. The vertical blue line marks the measured difference in amplitudes.

that the chemistry at both terminators is more similar than for WASP-76 b. An increase in the absorption line (which in this data may amount to a factor of 2, see Fig. 7) may be indicative of a scale-height difference on both terminators, however, which might be caused by the temperature on the evening terminator being higher than on the morning terminator.

\subsection{Summary}

We presented detections of atomic metal absorption lines in the transmission spectrum of the ultrahot Jupiter MASCARA2 b (Lund et al. 2017; Talens et al. 2018). To this end, one transit of MASCARA-2 b was observed with EXPRES, the high-resolution optical spectrograph newly commissioned at the Lowell Discovery Telescope. We confirm previous detections of Fe I, Fe II, and Na I (Casasayas-Barris et al. 2018, 2019; Nugroho et al. 2020; Stangret et al. 2020), and additionally find strong evidence for line absorption by atomic $\mathrm{Mg}$ I, and $\mathrm{Cr}$ II. All detected species appear to be blueshifted, indicating the presence of a dayto nightside wind, as has also been observed in previous studies (e.g., Nugroho et al. 2020; Stangret et al. 2020). Using the shape variation of the stellar absorption lines induced by the transiting planet (i.e., the Doppler shadow), we constrained the projected spin-orbit misalignment to $1.6 \pm 3.1$ degrees, consistent with an aligned orbit. The CCFs indicate time-variability in the absorption strength of these species, albeit at a level of $\lesssim 3 \sigma$. With a single transit, we cannot rule out that this variability is spurious. However, if it is astrophysical in origin, it might trace the differential atmospheric structure between morning and evening terminators, reminiscent of what has recently been observed in the transmission spectrum of WASP-76 b (Ehrenreich et al. 2020). These results demonstrate the first spectroscopic observation of an exoplanet atmosphere with the EXPRES instrument, demonstrating its future potential for atmospheric characterization. 
Acknowledgements. This work was supported by the PlanetS National Centre of Competence in Research (NCCR) supported by the Swiss National Science Foundation (SNSF), the NSF under grants NSF MRI-1429365 and ATI-1509436 and by the European Re- search Council (ERC) under the European Union's Horizon 2020 research and innovation program (projects Four Aces and EXOK LEIN with grant agreement numbers 724427 and 771620 , respectively). We acknowledge generous support for telescope time provided by the HeisingSimons Foundation and the Yale Astronomy Department. D.A.F. and J.M.B wish to acknowledge support from an anonymous donation, which has also been used for telescope time. L.L.Z. and A.B.D. gratefully acknowledge support from the NSF GRFP. These results made use of the Lowell Discovery Telescope at Lowell Observatory. Lowell is a private, non-profit institution dedicated to astrophysical research and public appreciation of astronomy and operates the LDT in partnership with Boston University, the University of Maryland, the University of Toledo, Northern Arizona University and Yale University. We thank the Lowell Observatory astronomers and staff for their extraordinary support.

\section{References}

Albrecht, S., Winn, J. N., Johnson, J. A., et al. 2012, ApJ, 757, 18

Allart, R., Lovis, C., Pino, L., et al. 2017, A\&A, 606, A144

Allart, R., Bourrier, V., Lovis, C., et al. 2019, A\&A, 623, A58

Astropy Collaboration (Robitaille, T. P., et al.) 2013, A\&A, 558, A33

Astropy Collaboration (Price-Whelan, A. M., et al.) 2018, AJ, 156, 123

Birkby, J. L. 2018, ArXiv e-prints [arXiv:1806. 04617]

Birkby, J. L., de Kok, R. J., Brogi, M., et al. 2013, MNRAS, 436, L35

Birkby, J. L., de Kok, R. J., Brogi, M., Schwarz, H., \& Snellen, I. A. G. 2017, AJ 153,138

Blackman, R. T., Ong, J. M. J., \& Fischer, D. A. 2019, AJ, 158, 40

Bourrier, V., Lecavelier des Etangs, A., Hébrard, G., et al. 2015, A\&A, 579, A55

Bourrier, V., Kitzmann, D., Kuntzer, T., et al. 2020, A\&A, 637, A36

Brogi, M., Snellen, I. A. G., de Kok, R. J., et al. 2012, Nature, 486, 502

Brogi, M., de Kok, R. J., Albrecht, S., et al. 2016, ApJ, 817, 106

Cabot, S. H. C., Madhusudhan, N., Hawker, G. A., \& Gandhi, S. 2019, MNRAS, 482,4422

Casasayas-Barris, N., Pallé, E., Yan, F., et al. 2018, A\&A, 616, A151

Casasayas-Barris, N., Pallé, E., Yan, F., et al. 2019, A\&A, 628, A9

Cauley, P. W., Shkolnik, E. L., Ilyin, I., et al. 2019, AJ, 157, 69

Cegla, H. M., Lovis, C., Bourrier, V., et al. 2016, A\&A, 588, A127

Claudi, R., Benatti, S., Carleo, I., et al. 2017, Eur. Phys. J. Plus, 132, 364

Collier Cameron, A., Bruce, V. A., Miller, G. R. M., Triaud, A. H. M. J., \& Queloz, D. 2010, MNRAS, 403, 151

Cosentino, R., Lovis, C., Pepe, F., et al. 2012, SPIE Conf. Ser., 8446, 84461V

Crossfield, I. J. M. 2015, PASP, 127, 941

Delrez, L., Santerne, A., Almenara, J. M., et al. 2016, MNRAS, 458, 4025

Ehrenreich, D., Lovis, C., Allart, R., et al. 2020, Nature, 580, 597

Evans, T. M., Sing, D. K., Goyal, J. M., et al. 2018, AJ, 156, 283

Fischer, D. A., Anglada-Escude, G., Arriagada, P., et al. 2016, PASP, 128, 066001

Flagg, L., Johns-Krull, C. M., Nofi, L., et al. 2019, ApJ, 878, L37

Foreman-Mackey, D., Hogg, D. W., Lang, D., \& Goodman, J. 2013, PASP, 125, 306

Gaudi, B. S., Stassun, K. G., Collins, K. A., et al. 2017, Nature, 546, 514
Grimm, S. L., \& Heng, K. 2015, ApJ, 808, 182

Guilluy, G., Sozzetti, A., Brogi, M., et al. 2019, A\&A, 625, A107

Hawker, G. A., Madhusudhan, N., Cabot, S. H. C., \& Gandhi, S. 2018, ApJ, 863, L11

Heng, K., \& Showman, A. P. 2015, Ann. Rev. Earth Planet. Sci., 43, 509

Hoeijmakers, H. J., Ehrenreich, D., Heng, K., et al. 2018a, Nature, 560, 453

Hoeijmakers, H. J., Snellen, I. A. G., \& van Terwisga, S. E. 2018b, A\&A, 610, A47

Hoeijmakers, H. J., Ehrenreich, D., Kitzmann, D., et al. 2019, A\&A, 627, A165

Husser, T.-O., Wende-von Berg, S., Dreizler, S., et al. 2013, A\&A, 553, A6

Jensen, A. G., Redfield, S., Endl, M., et al. 2012, ApJ, 751, 86

Khalafinejad, S., von Essen, C., Hoeijmakers, H. J., et al. 2017, A\&A, 598, A131

Levine, S. E., Bida, T. A., Chylek, T., et al. 2012, SPIE Conf. Ser., 8444, 844419

Lockwood, A. C., Johnson, J. A., Bender, C. F., et al. 2014, ApJ, 783, L29

Louden, T., \& Wheatley, P. J. 2015, ApJ, 814, L24

Lund, M. B., Rodriguez, J. E., Zhou, G., et al. 2017, AJ, 154, 194

Madhusudhan, N., Agúndez, M., Moses, J. I., \& Hu, Y. 2016, Space Sci. Rev., 205,285

Nugroho, S. K., Kawahara, H., Masuda, K., et al. 2017, AJ, 154, 221

Nugroho, S. K., Gibson, N. P., de Mooij, E. J. W., et al. 2020, MNRAS, submitted [arXiv:2003.04856]

Ohta, Y., Taruya, A., \& Suto, Y. 2005, ApJ, 622, 1118

Oliva, E., Sanna, N., Rainer, M., et al. 2018, SPIE, 10701, 2118

Pepe, F., Cristiani, S., Rebolo, R., et al. 2013, The Messenger, 153, 6

Petersburg, R. R., Joel Ong, J. M., Zhao, L. L., et al. 2020, AJ, 159, 187

Pino, L., Ehrenreich, D., Allart, R., et al. 2018, A\&A, 619, A3

Piskorz, D., Benneke, B., Crockett, N. R., et al. 2016, ApJ, 832, 131

Quirrenbach, A., Amado, P. J., Mandel, H., et al. 2010, SPIE Conf. Ser., 7735, 773513

Redfield, S., Endl, M., Cochran, W. D., \& Koesterke, L. 2008, ApJ, 673, L87

Schlaufman, K. C. 2010, ApJ, 719, 602

Seidel, J. V., Ehrenreich, D., Wyttenbach, A., et al. 2019, A\&A, 623, A166

Showman, A. P., Fortney, J. J., Lewis, N. K., \& Shabram, M. 2013, ApJ, 762, 24

Smette, A., Sana, H., Noll, S., et al. 2015, A\&A, 576, A77

Snellen, I. A. G., Albrecht, S., de Mooij, E. J. W., \& Le Poole, R. S. 2008, A\&A, 487,357

Snellen, I. A. G., de Kok, R. J., de Mooij, E. J. W., \& Albrecht, S. 2010, Nature, 465, 1049

Stangret, M., Casasayas-Barris, N., Pallé, E., et al. 2020, A\&A, 638, A26

Stock, J. W., Kitzmann, D., Patzer, A. B. C., \& Sedlmayr, E. 2018, MNRAS, 479, 865

Talens, G. J. J., Justesen, A. B., Albrecht, S., et al. 2018, A\&A, 612, A57

Thibault, S., Rabou, P., Donati, J.-F., et al. 2012, Proc. SPIE, 8446, 844630

Thompson, S. J., Queloz, D., Baraffe, I., et al. 2016, SPIE Conf. Ser., 9908, 99086F

Triaud, A. H. M. J. 2018, Handbook of Exoplanets (Cham: Springer), 2

Turner, J. D., de Mooij, E. J. W., Jayawardhana, R., et al. 2020, ApJ, 888, L13

Wildi, F., Blind, N., Reshetov, V., et al. 2017, SPIE Conf. Ser., 10400, 1040018

Winn, J. N., Fabrycky, D., Albrecht, S., \& Johnson, J. A. 2010, ApJ, 718, L145

Wright, J. T. 2018, Handbook of Exoplanets (Cham: Springer), 4

Wyttenbach, A., Ehrenreich, D., Lovis, C., Udry, S., \& Pepe, F. 2015, A\&A, 577, A62

Wyttenbach, A., Lovis, C., Ehrenreich, D., et al. 2017, A\&A, 602, A36

Yan, F., \& Henning, T. 2018, Nat. Astron., 2, 714 


\section{Appendix A: MCMC results and additional figures}

Table A.1. MCMC results for modeling of the RM effect.

\begin{tabular}{lccrrr}
\hline \hline Parameter & Symbol & Unit & MCMC median & T18 (prior) & L17 \\
\hline Scaled semimajor axis & $a / R_{*}$ & - & $7.50 \pm 0.04$ & $7.50 \pm 0.04$ & $7.44_{-0.13}^{+0.14}$ \\
Projected obliquity & $\lambda$ & $\circ$ & $1.58_{-3.12}^{+3.14}$ & $0.6 \pm 4$ & $3.4 \pm 2.1$ \\
Orbit inclination & $i_{p}$ & $\circ$ & $86.16 \pm 0.5$ & $86.4_{-0.4}^{+0.5}$ & $86.15_{-0.27}^{+0.28}$ \\
Projected stellar rotation speed & $v \sin i_{*}$ & $\mathrm{~km} \mathrm{~s}^{-1}$ & $113.50_{-0.74}^{+0.77}$ & $114 \pm 3$ & $115.9 \pm 3.4$ \\
RV offset & - & $\mathrm{km} \mathrm{s}^{-1}$ & $0.7 \pm 3.1$ & $0 \pm 5^{*}$ & - \\
\hline
\end{tabular}

Notes. The model consists of the four parameters listed in Col. 1, plus a global RV offset. The MCMC median values are listed in Col. 4. Uncertainties correspond to the 16th and 84th percentiles. For comparison, we list literature values in Col. 5, and their references in Col. 6. T18 denotes Talens et al. (2018), and L17 denotes Lund et al. (2017). The asterisk indicates an RV offset prior that is not taken from the literature.

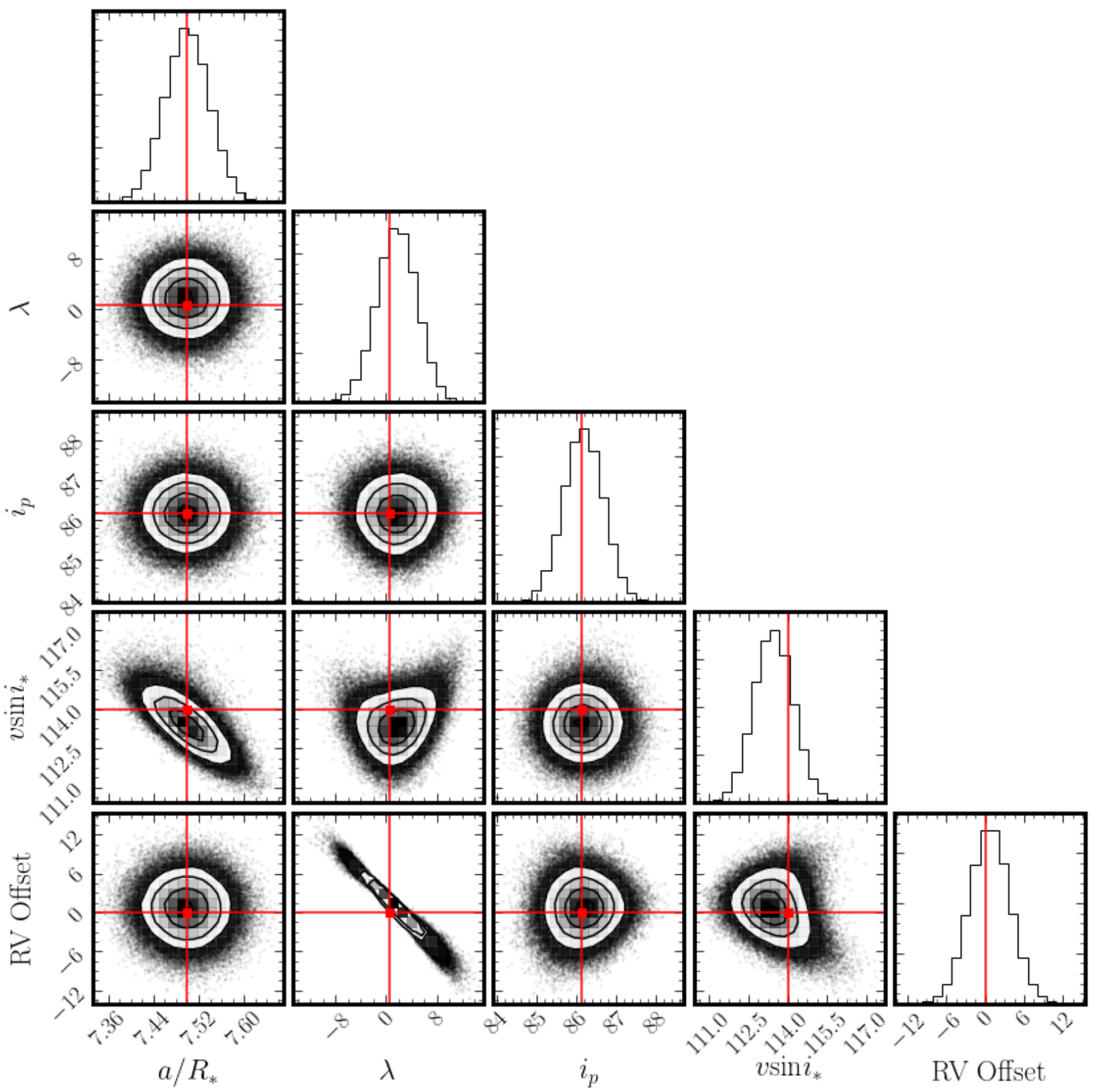

Fig. A.1. Correlation diagram from our MCMC fit to the observed Doppler shadow, as well as 1D histograms. The model consists of five parameters: scaled semimajor axis $\left(a / R_{*}\right)$, projected obliquity $(\lambda)$, orbital inclination $\left(i_{p}\right)$, projected stellar rotation speed $\left(v \sin i_{*}\right)$, and a global RV offset. Red lines denote literature values for the parameters (Talens et al. 2018). 

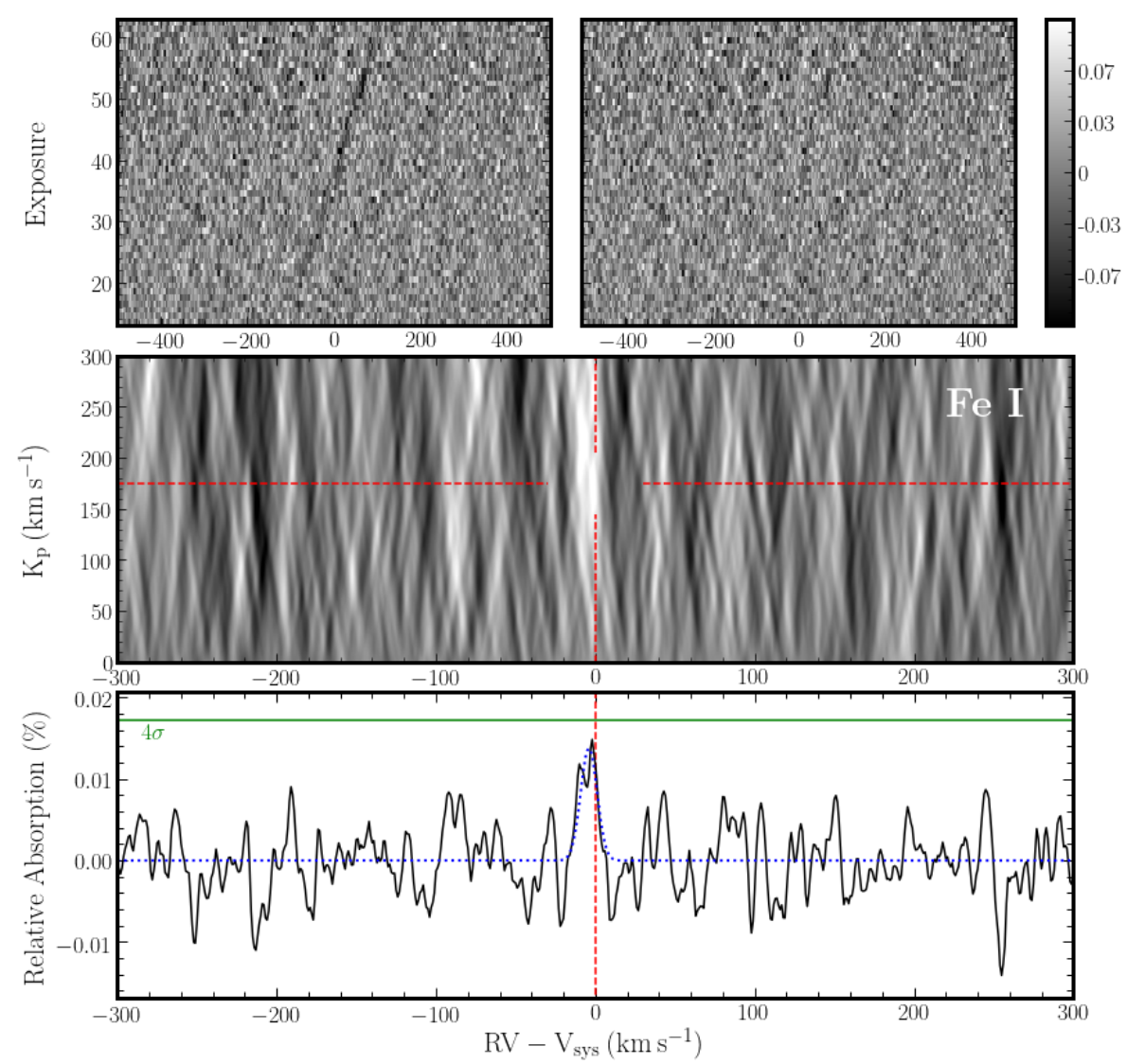

Fig. A.2. Same as Fig. 4, depicting the cross-correlation process for Fe I. CCFs are in the rest frame of the planet.

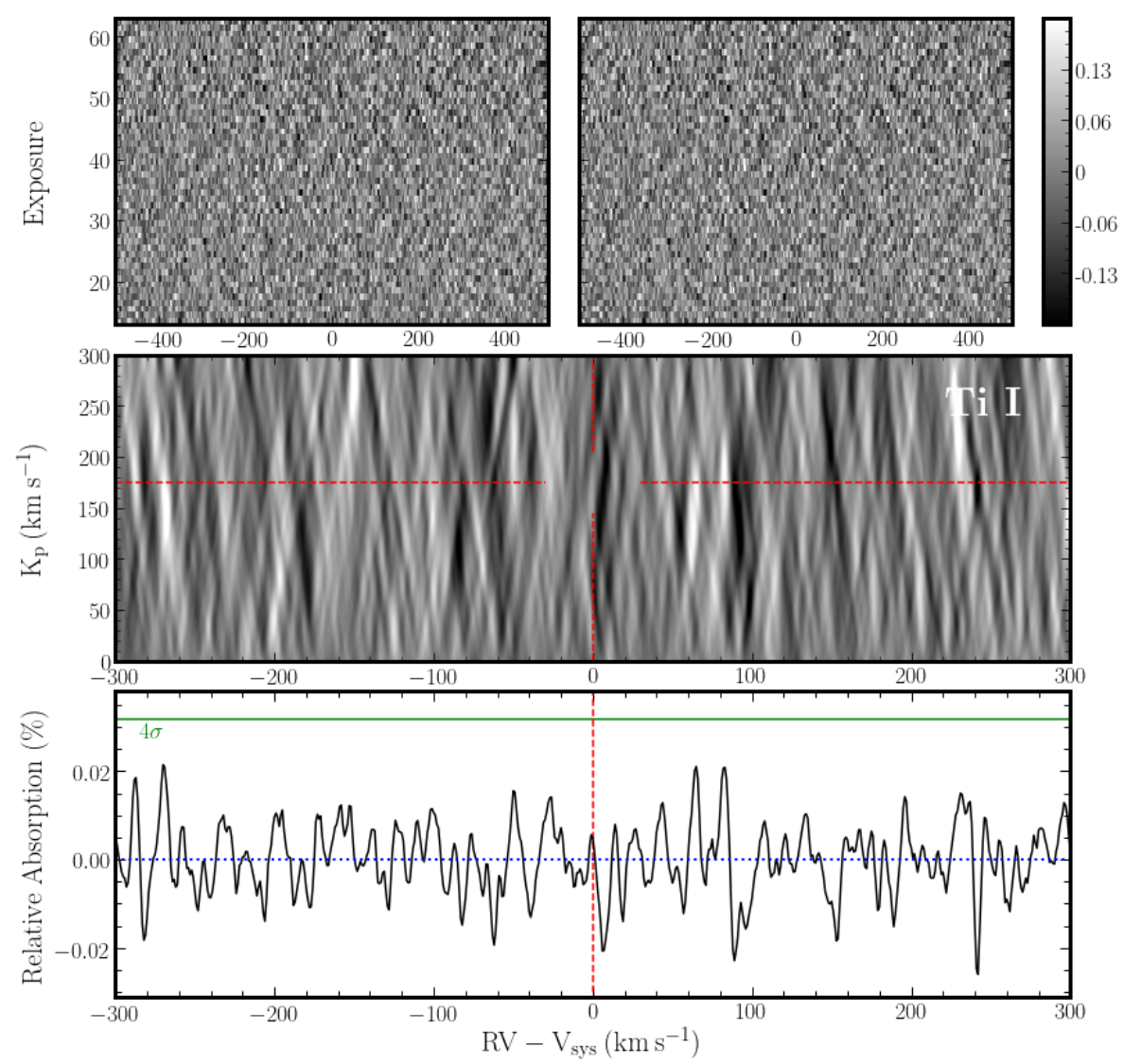

Fig. A.3. Same as Fig. 4, depicting the cross-correlation process for Ti I. CCFs are in the rest frame of the planet. 
H. J. Hoeijmakers et al.: EXPRES transmission spectroscopy of MASCARA-2 b
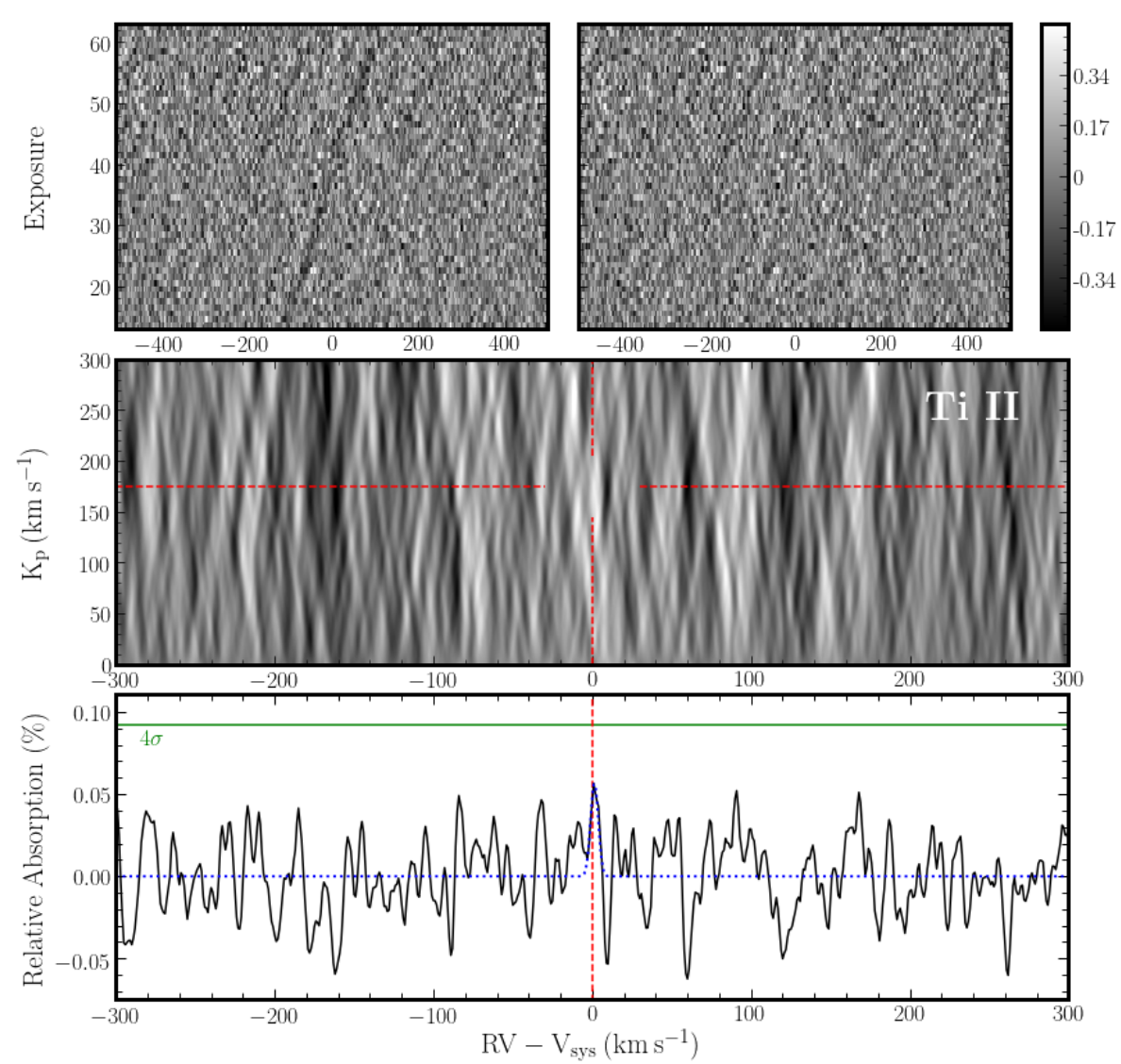

Fig. A.4. Same as Fig. 4, depicting the cross-correlation process for Ti II. CCFs are in the rest frame of the planet.
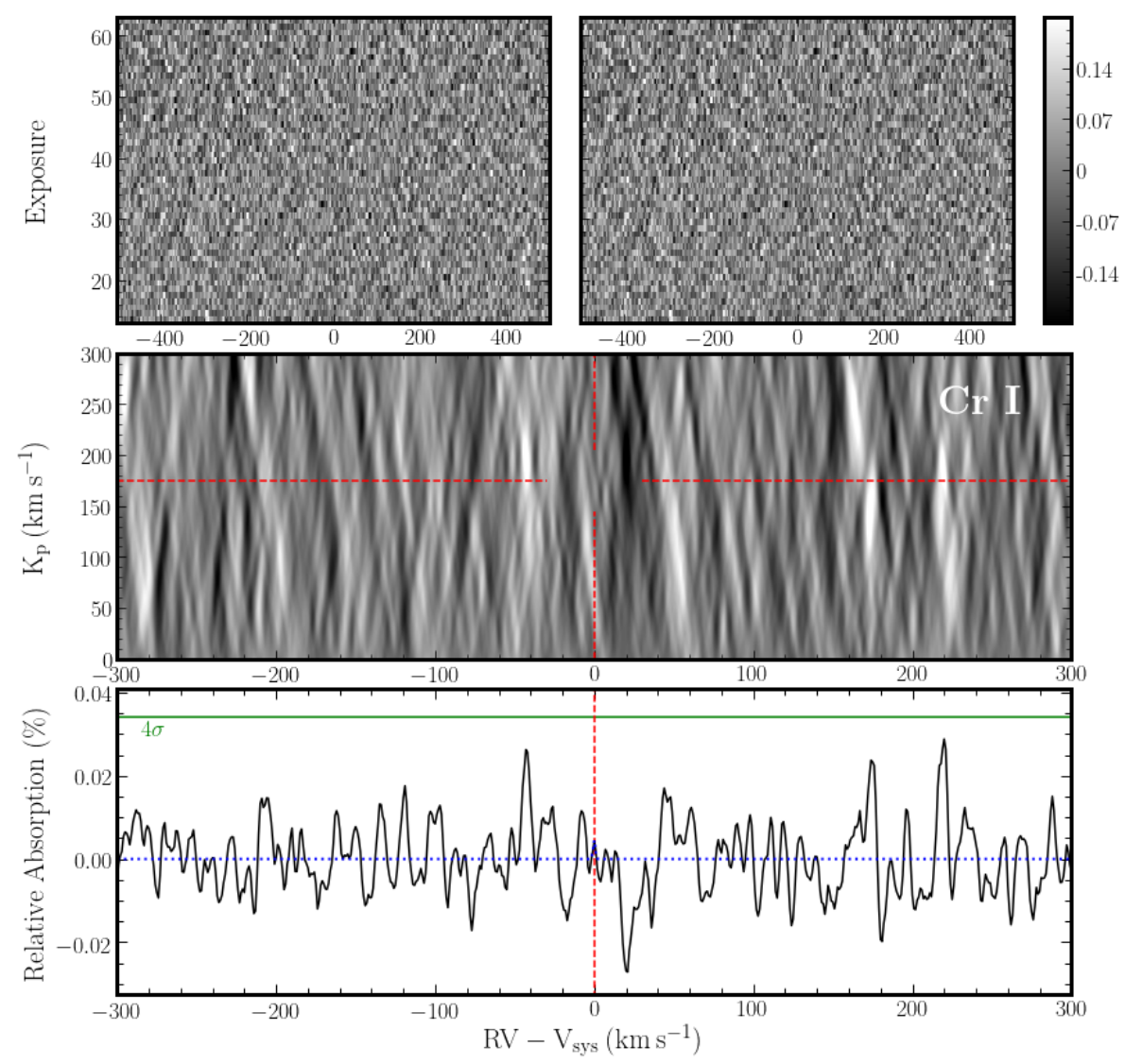

Fig. A.5. Same as Fig. 4, depicting the cross-correlation process for Cr I. CCFs are in the rest frame of the planet. 

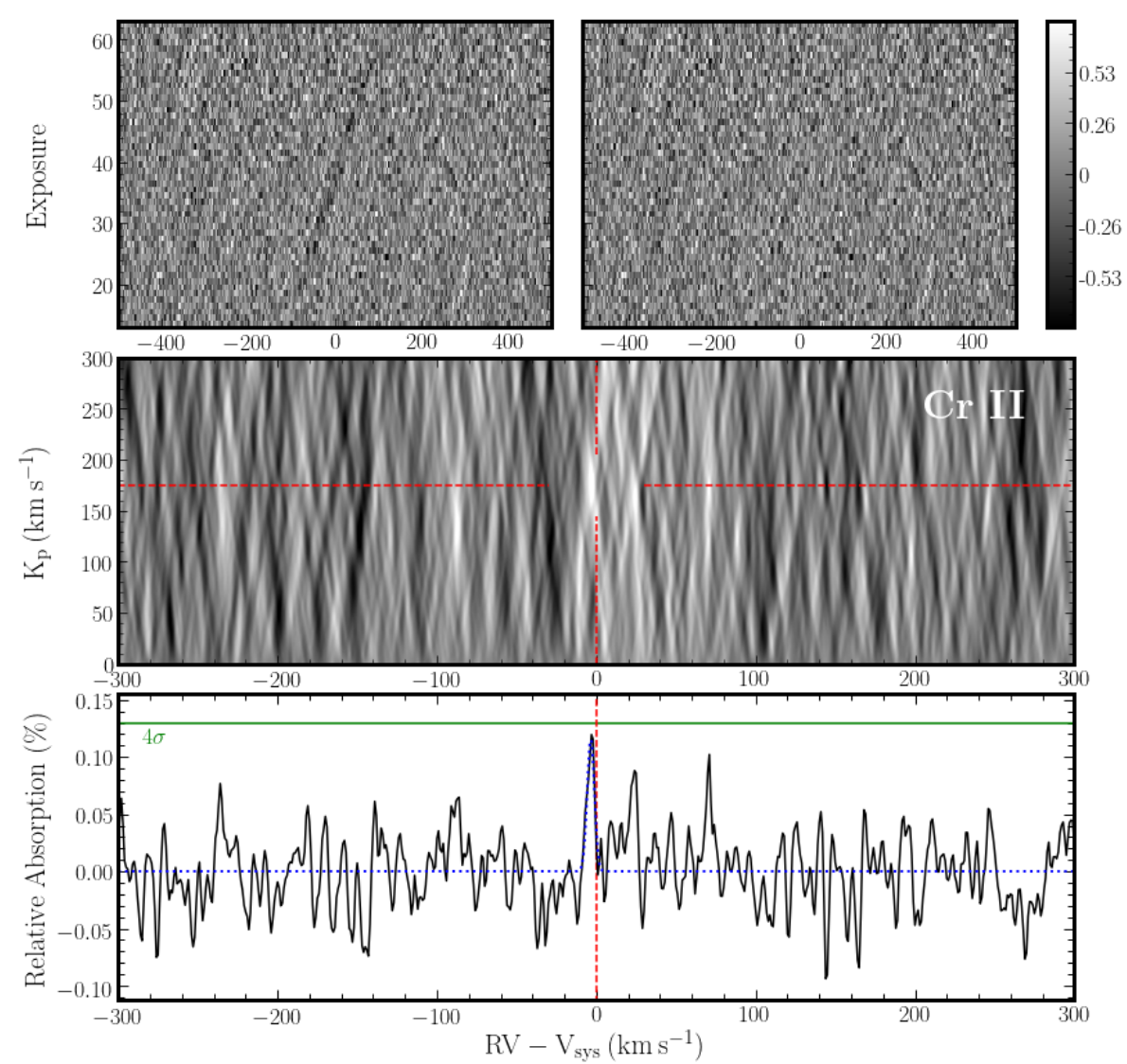

Fig. A.6. Same as Fig. 4, depicting the cross-correlation process for Cr II. CCFs are in the rest frame of the planet.
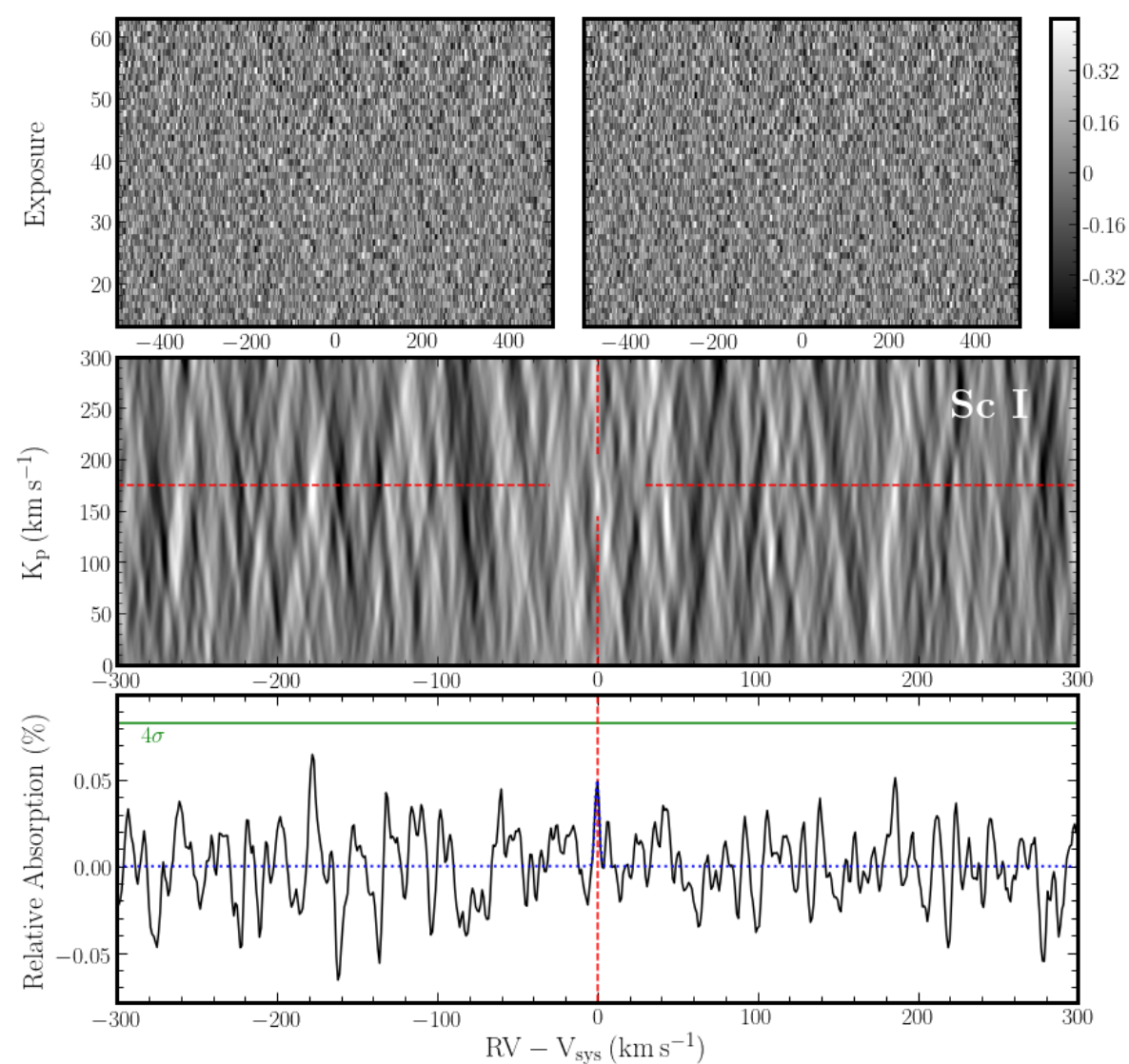

Fig. A.7. Same as Fig. 4, depicting the cross-correlation process for Sc I. CCFs are in the rest frame of the planet. 
H. J. Hoeijmakers et al.: EXPRES transmission spectroscopy of MASCARA-2 b
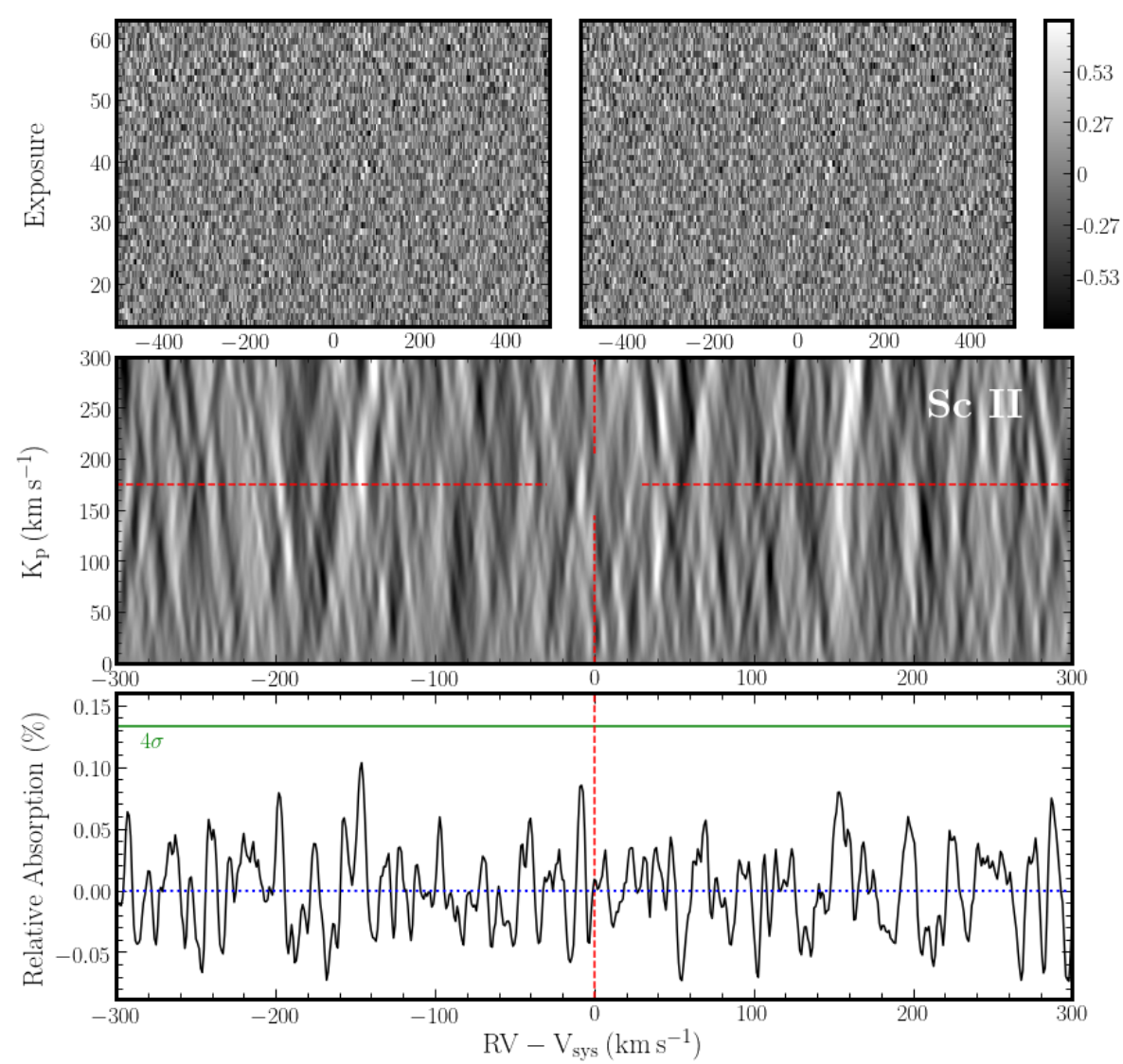

Fig. A.8. Same as Fig. 4, depicting the cross-correlation process for Sc II. CCFs are in the rest frame of the planet.
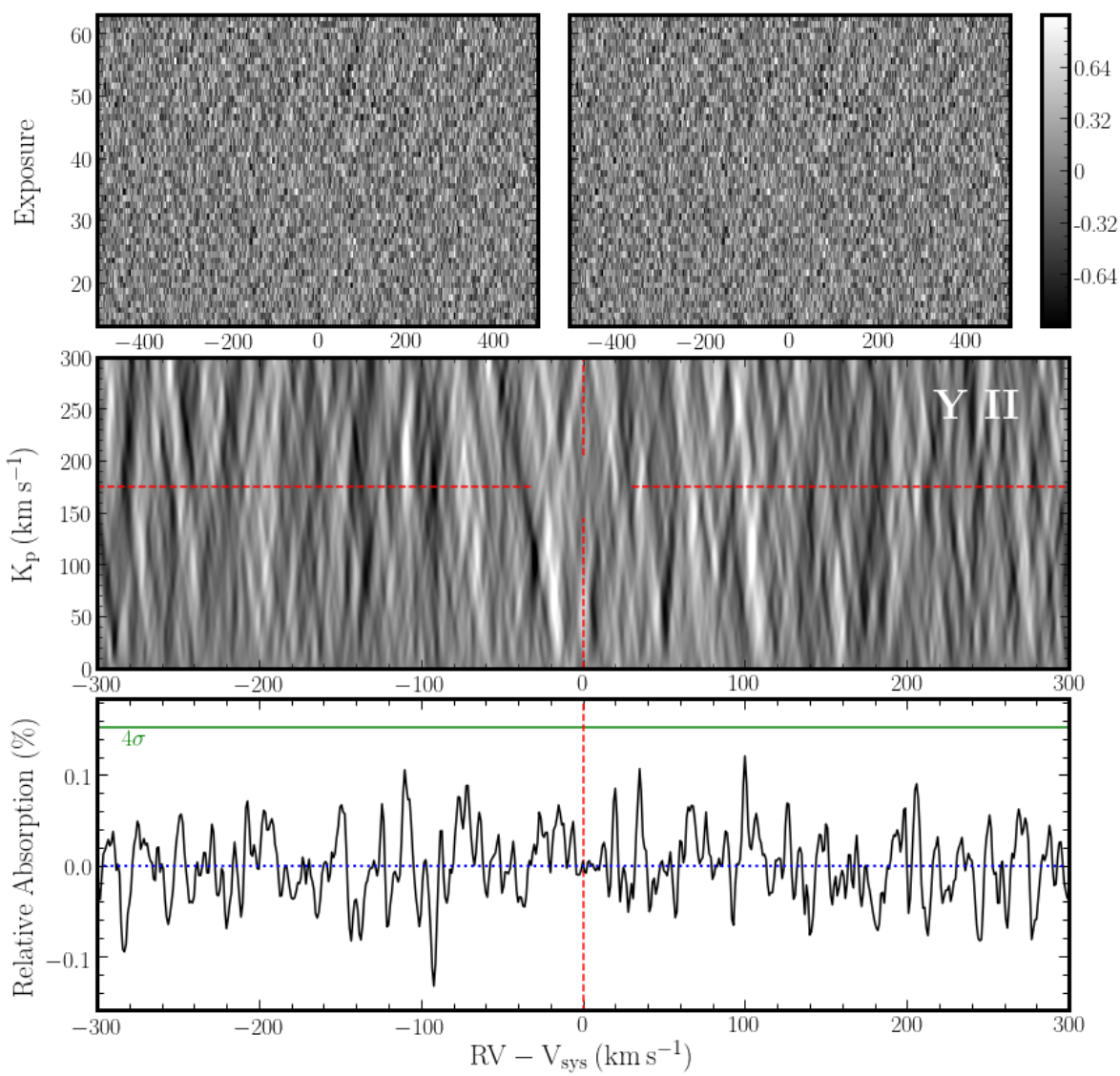

Fig. A.9. Same as Fig. 4, depicting the cross-correlation process for Y II. CCFs are in the rest frame of the planet. 

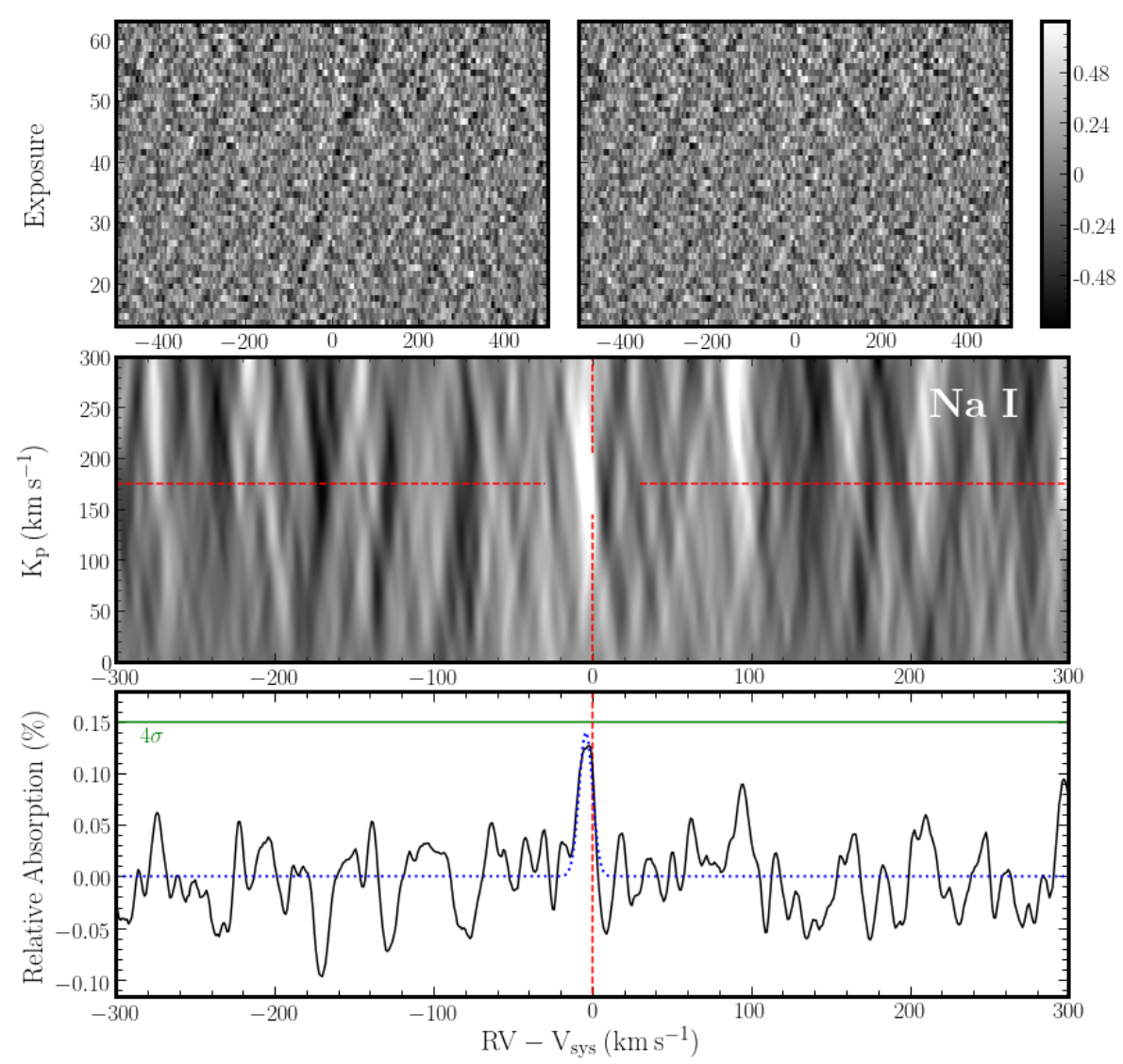

Fig. A.10. Same as Fig. 4, depicting the cross-correlation process for Na I. CCFs are in the rest frame of the planet.
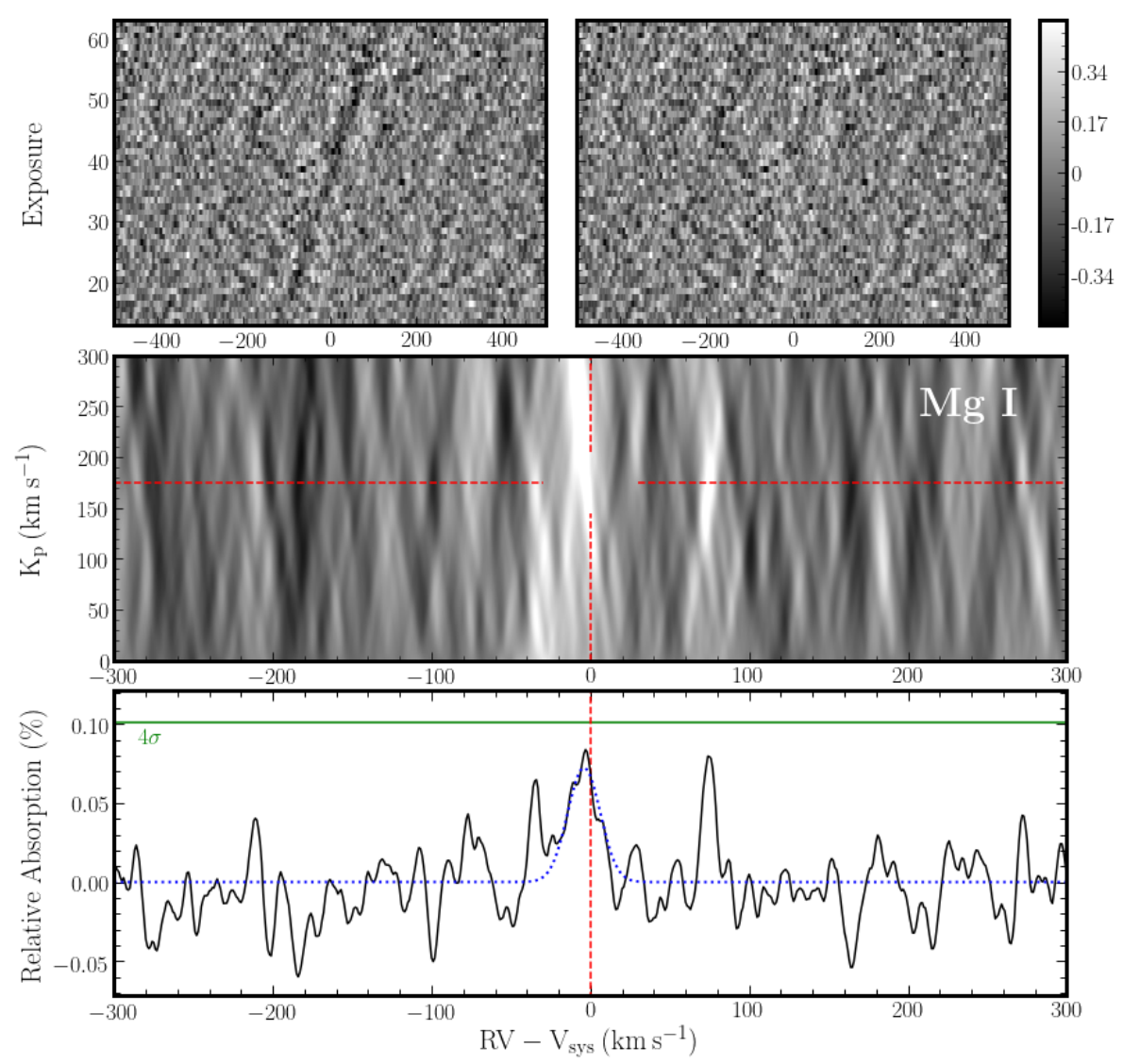

Fig. A.11. Same as Fig. 4, depicting the cross-correlation process for Mg I. CCFs are in the rest frame of the planet. 

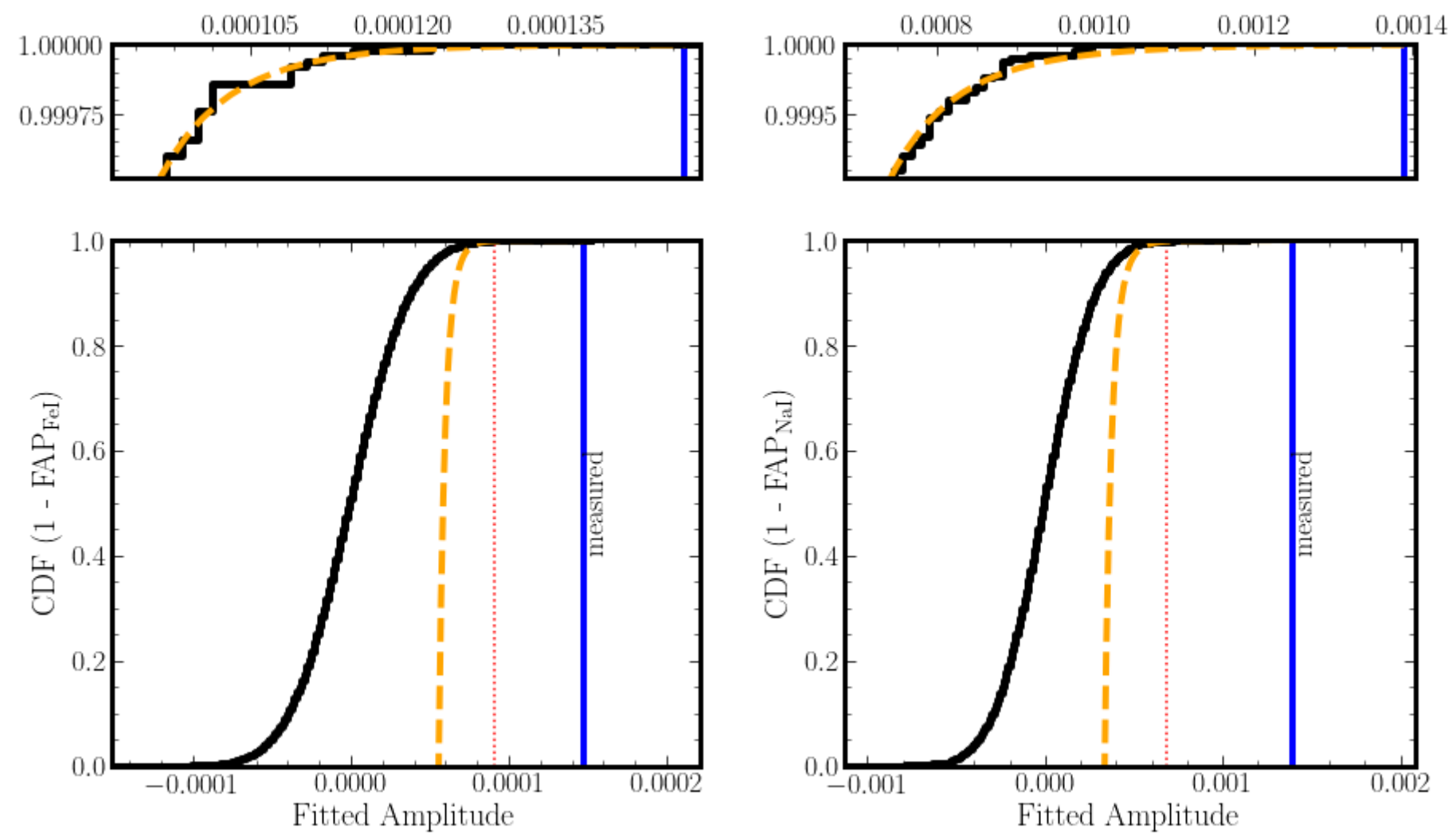

Fig. A.12. Same as Fig 5, except based on cross-correlation with the Fe I template. This plot shows the distribution of strengths of random signals generated by the CCFs, from which the FAP is derived.

Fig. A.14. Same as Fig 5, except based on cross-correlation with the $\mathrm{Na}$ I template. This plot shows the distribution of strengths of random signals generated by the CCFs, from which the FAP is derived.
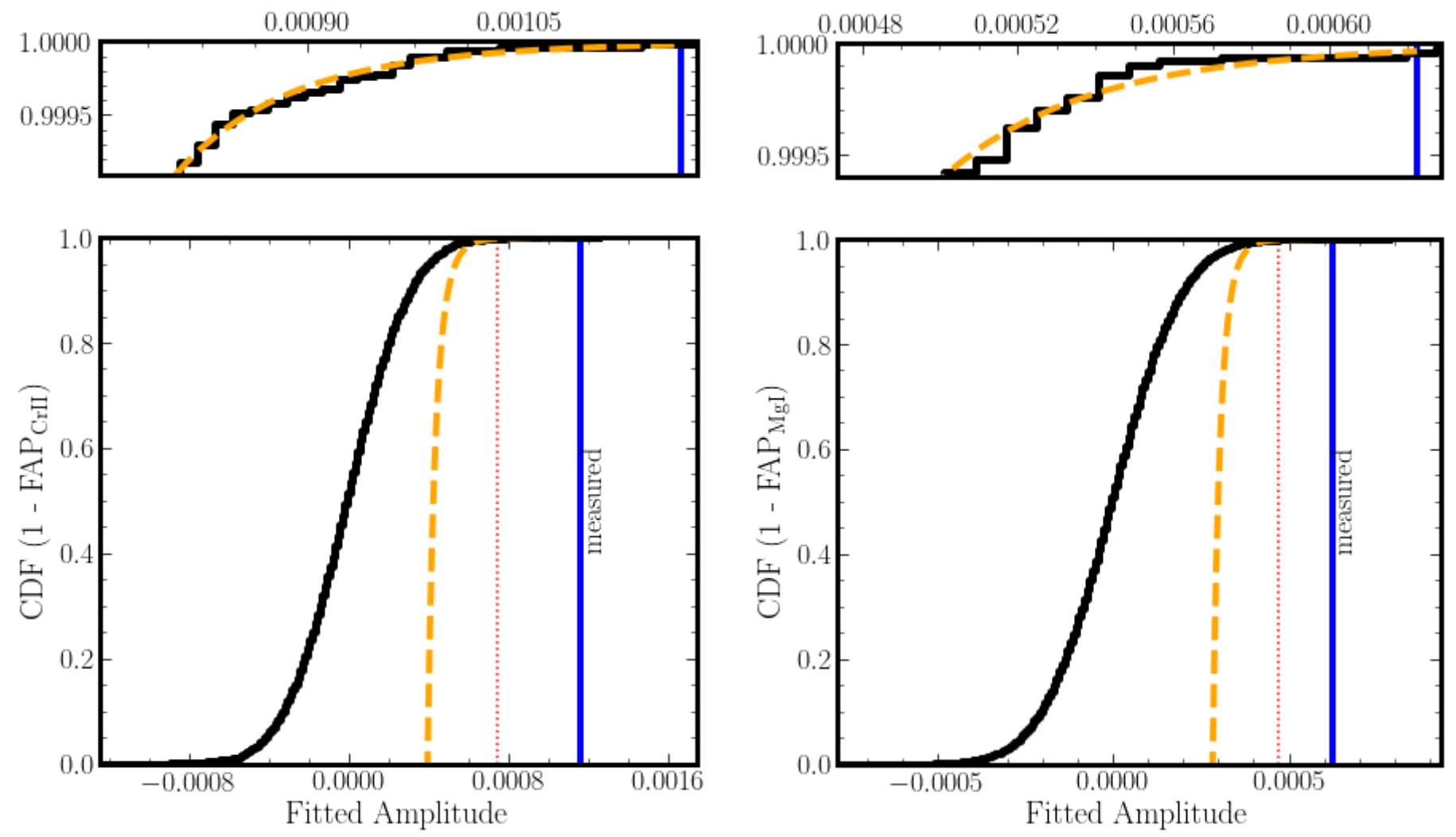

Fig. A.13. Same as Fig 5, except based on cross-correlation with the Cr II template. This plot shows the distribution of strengths of random signals generated by the CCFs, from which the FAP is derived.

Fig. A.15. Same as Fig 5, except based on cross-correlation with the $\mathrm{Mg}$ I template. This plot shows the distribution of strengths of random signals generated by the CCFs, from which the FAP is derived. 